\title{
Gelation Kinetics of Non-Aqueous Carbopol Dispersions
}

\author{
Simona Migliozzi, Panagiota Angeli* and Luca Mazzei* \\ Department of Chemical Engineering, University College London, Torrington Place, London WC1E 7JE, UK \\ *Corresponding authors: p.angeli@ucl.ac.uk; l.mazzei@ucl.ac.uk
}

\begin{abstract}
The time evolution of the gelation process of non-aqueous Carbopol dispersions in PEG and glycerol was studied experimentally by tracking the evolving viscoelastic properties of the material by means of Fourier Transform Mechanical Spectroscopy (FTMS). A structural conversion degree related to the storage modulus was defined and a kinetic expression to describe its evolution was developed. UV-vis spectroscopy was employed to confirm the mechanism of gelation. It was found that an increase in both the concentration of glycerol and the gelation temperature increased the gelation rate. These trends seem to indicate a diffusion-controlled mechanism of the process, in which the solvent molecules penetrate the cross-linked structure of Carbopol particles, causing their uncoiling and subsequent swelling. Lastly, the kinetic relation found for the structural conversion degree was used to obtain conversion maps, which can be employed as a first guidance to design new continuous gelation formulation processes.
\end{abstract}

Keywords: time-resolved rheometry, gelation kinetics, Carbopol gels, UV-vis spectroscopy.

\section{Introduction}

The process of gelation can be described as the conversion of a liquid solution into a medium with a solid-like behaviour, constituted by an internal network of molecules, assembled by means of chemical or physical bonds to form a complex structure. Specifically, polymeric gels consist of cross-linked solutions of polymer blends which form either by direct cross- 
linking of polymer chains in a liquid diluent or by swelling of already cross-linked chains in a solvent. In the first case, the diluent is used as the liquid matrix in which the polymer chains start to react and cross-link both among themselves and with the liquid diluent molecules. In the second case, the surrounding liquid acts exclusively as a solvent, making the already cross-linked chains of polymer swell, involving purely physical interactions [1,2]. Typical examples of molecules that create swollen gels when dissolved in hydrophilic solvents are Carbopols. These commercial molecules are high molecular weight polymers of polyacrylic acid usually cross-linked with allyl sucrose $[3,4]$. Thanks to their synthetic nature and high thickening properties, these polymers have been widely employed in an increasing number of industrial (e.g., cosmetics, food processing and pharmaceuticals) and research (e.g., targeted drug delivery and tissue engineering) applications. Water is the most common solvent used as base for Carbopol gels preparations. Different mechanisms have been proposed over the years to model the gelation process [3]. For the case of polar solvents, such as water, the most accredited theory considers the dissociation of the polyacrylic acid groups. Usually, a base (e.g., sodium hydroxide or triethanolamine) is added during the process to neutralise the acidic solution causing the ionization of the carboxylate groups. Consequently, uncompensated ions lead to an increase in the mutual ionic repulsion inside the polymer chain causing a dramatic swelling of each polymer particle, which eventually results into complete packing. The final gel is then constituted of a micro-gel system that behaves like an elastic solid up to a certain threshold value of applied stress at which the system breaks down (yield stress) [4-9].

Less is known about the behaviour of Carbopol gels obtained in other solvents. The use of alternative non-aqueous solvents, usually partially organic, appears to be necessary in different pharmaceutical applications, when water insoluble drugs need to be added to the gelled system. In this case, in the absence of a neutralising agent, solvent molecules penetrate 
into the cross-linked structures and uncoil them by creating hydrogen bonds with the lateral carboxyl groups of the polymer chains, thus making them retain their final swelled conformation [3]. The effect of a variety of solvent mixtures on the final properties of many Carbopol molecules has been studied by some investigators [10-12], but to the knowledge of the authors there is no study focusing on the kinetic aspects of the gelation process. Indeed, a quantitative analysis of the time evolution of the material structure is of paramount importance for process design applications and scale-up, especially if a dramatic change of the material flow properties is expected.

In the literature, the formation of Carbopol gels, and more in general of polymer gels, appears to be influenced by three core aspects: solvent composition, operating temperature and shear history $[9,13-15]$. The aim of this work is to obtain a kinetic analysis of the evolution of the structure of non-aqueous Carbopol dispersions with focus on the first two aspects: solvent composition and operating temperature; the role of shear rate, although crucial for a direct translation into industrial processes, was not considered at this stage to obtain a first insight into the mechanism of gelation independently from the presence of applied flow fields. Specifically, samples with various compositions were prepared using two different solvents, polyethylene glycol (PEG400) and glycerol. Both solvents are commonly used in different pharmaceutical and healthcare product formulations in which it is crucial to dissolve water insoluble drugs into a hydrophilic and biocompatible matrix, e.g. drug release, toothpaste, ophthalmic applications [16,17]. Carbopol was added to a fixed amount of PEG400 and then diluted with different amounts of glycerol. This procedure was implemented to investigate what the effect of diluting the initial Carbopol/PEG mixture with glycerol would be during a mixing/gelation coupled process.

A Fourier Transform Mechanical Spectroscopy (FTMS) technique, known as multi-wave analysis, was employed to track the evolving properties of the tested samples and obtain a 
macroscopic description of the kinetics of gelation. UV-vis spectroscopy was also used to obtain gelation times for comparison and to confirm the main conclusions obtained from the rheological tests on the mechanism of gelation.

The article is organized as follows. Materials and experimental methods are introduced first; then, we present the criteria used to characterize the gelation transition, followed by results and comments on the experimental findings. Subsequently, a mechanism for the gelation process is suggested. To conclude, we report conversion maps which can be used as a tool for a first-step design of the gelation process.

\section{Materials and Experimental Methods}

\subsection{Materials}

Carbopol 974P NF (Lubrizol Limited) in powder form was used to prepare a polymeric dispersion in Polyethylene glycol 400 (PEG400, Ineos Oxide). This type of Carbopol is a highly-crosslinked polymer of acrylic acid cross-linked with allyl pentaerythritol [18], although specifications on the crosslinking process for Carbopol 974P NF are not known. The dispersion was prepared by adding 4\% wt of Carbopol in PEG400. A high-shear mixer (Silverson, L5 Series) working at $7000 \mathrm{rpm}$ was used to disperse homogeneously the polymer into the liquid phase. During the dispersion step, the beaker was placed in a water/ice bath, and the temperature of the mixture, kept in the range between 3 and $5{ }^{\circ} \mathrm{C}$, was constantly controlled with a digital thermometer. It is important to keep the mixture temperature below $20{ }^{\circ} \mathrm{C}$ to prevent the initiation of the gelation process during the dispersion step. The dispersion was mixed for approximately 15 minutes. After preparation, the homogenized dispersion was stored at $5^{\circ} \mathrm{C}$ for 30 minutes. This stage is essential to quench the heat produced in the liquid bulk by the high friction of the mixer. The final samples were prepared in batches of $5 \mathrm{ml}$ by adding pure Glycerol (Avantor Performance Materials, Inc.) in different 
amounts to the polymeric dispersion and by mixing the small liquid volumes by hand for about 5 seconds just before loading the instruments. The usual temperature of the mixtures before loading was around $15^{\circ} \mathrm{C}$. The final volume of mixture loaded for all the experimental measurements was $1 \mathrm{ml}$. The room temperature during all measurements was $\sim 21^{\circ} \mathrm{C}$.

\subsection{Fourier Transform Mechanical Spectroscopy}

The viscoelastic properties of the gelling material were tracked in time using a time-resolved rheometry technique known as multi-wave analysis. Commonly, it is necessary to obtain the frequency dependence of both elastic and viscous moduli to draw a complete picture of the internal structure of a complex fluid. Hence, if the tested material is subjected to a structural change in time, the method most commonly used to record its evolution is to stop the process which is causing the internal changes and perform a frequency sweep at different stages of the material evolution [13,19-21]. However, in some cases full control of the process is not feasible and consequently the microstructure evolution cannot be paused as needed. Furthermore, this technique yields too coarse a description of the time evolution of the material viscoelastic properties, and a more "continuous" description of the changes in the internal structure of the material is desirable. To this end, it is more appropriate to apply a periodic sinusoidal deformation, characterized by a specific amplitude and oscillation frequency, and observe how the storage and loss moduli evolve in time. In this case, the same experiment should be repeated with different oscillation frequencies to obtain a complete picture of the structure evolution. FTMS methods offer the advantage to extract the time evolution of the viscoelastic properties at several frequencies with a single experiment. This technique, introduced by Holly et al. [22] and adopted by various researchers [23-25], consists in applying a periodic shear deformation given by the summation of different harmonics of a base sinusoid: 


$$
\gamma(t)=\sum_{i=1}^{m} a_{i} \gamma_{0} \sin \left(k_{i} \omega_{0} t\right)
$$

where $\mathrm{m}$ is the number of harmonics, while $a_{i}$ and $k_{i}$ are scale factors for the deformation amplitude $\gamma_{0}$ and frequency $\omega_{0}$ of the base sinusoid, respectively.

An example of the deformation function $\gamma(t)$ is shown in Figure 1.

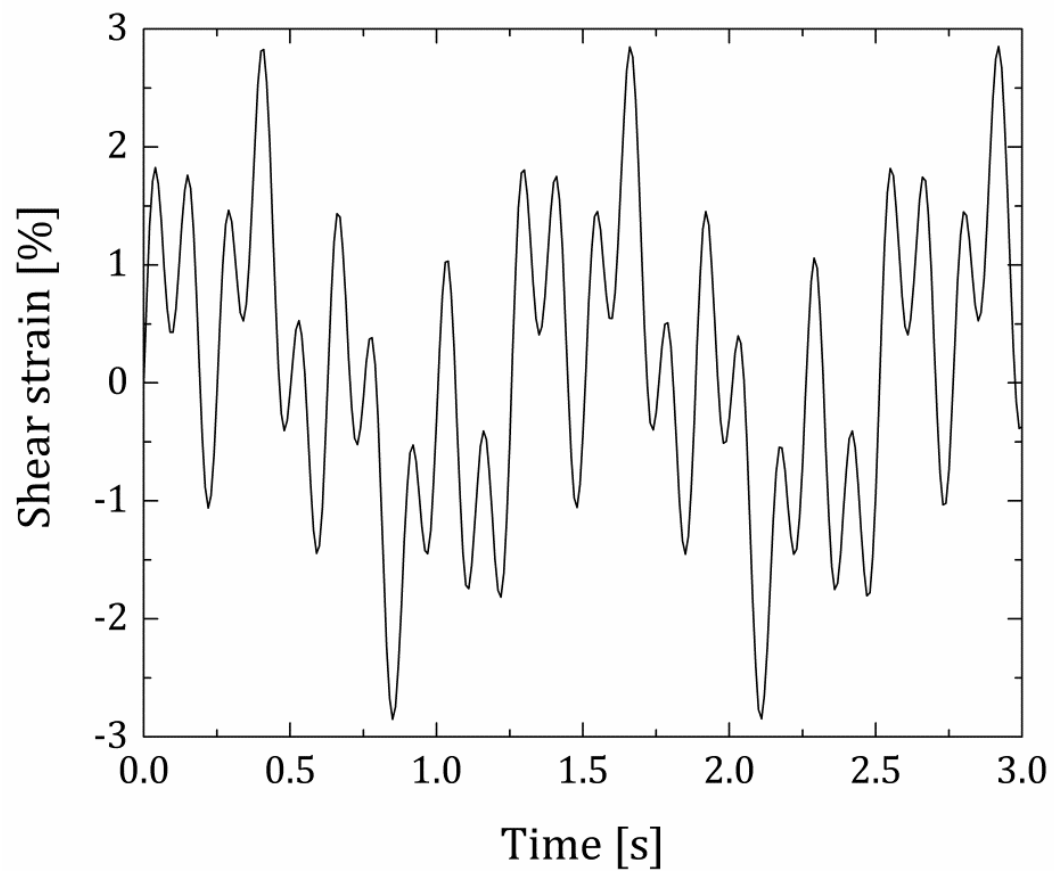

Figure 1 - Example of shear deformation applied in a multi-wave analysis.

If the maximum amplitude of the deformation is in the linear viscoelastic region (LVR), then the storage modulus $G^{\prime}$ and the loss modulus $G^{\prime \prime}$, related to the elastic component and the viscous component of the oscillatory shear stress [1] (see SI-1 for further details), respectively, can be directly obtained from a Fast Fourier Transform (FFT) analysis of the shear deformation $\gamma(t)$ and of the corresponding shear stress $\sigma(t)$ recorded. Through this analysis the contribution of each harmonic can be isolated from the others and the time evolution of the storage modulus $G^{\prime}$ and the loss modulus $G^{\prime \prime}$ at each frequency can be obtained as follows: 


$$
\begin{aligned}
& G^{\prime}\left(\omega_{i}, t\right)=\operatorname{Re}\left[\frac{F F T(\sigma(t))}{F F T(\gamma(t))}\right] \\
& G^{\prime \prime}\left(\omega_{i}, t\right)=\operatorname{Im}\left[\frac{F F T(\sigma(t))}{F F T(\gamma(t))}\right]
\end{aligned}
$$

where $\omega_{i}$ is the oscillation frequency of a single harmonic, given by the product between $k_{i}$ and $\omega_{0}$ (Eq.1), $\sigma(t)$ is the recorded shear stress signal and finally Re and Im are the real and imaginary parts of the Fast Fourier transform, respectively [26]. Then, $\tan \delta$ is given by the ratio $G^{\prime \prime} / G^{\prime}[27]$.

Table 1 - Parameter values of the sinusoidal shear deformation applied for the FTMS experiments.

\begin{tabular}{cc}
\hline PARAMETERS & VALUES \\
\hline \hline$\gamma_{0}$ & $1 \%$ \\
$\omega_{0}$ & $1 \mathrm{rad} / \mathrm{s}$ \\
$\boldsymbol{a}_{\boldsymbol{i}}$ & 1 \\
$\boldsymbol{k}_{\boldsymbol{i}}$ & $1,5,20,50$ \\
\hline
\end{tabular}

The TA Instruments DHR3 was used to carry out all the rheological measurements. This is a stress-controlled rotational rheometer, equipped with a Peltier plate to precisely control the operating temperature. We used a stainless steel, sandblasted parallel plate geometry (with surface roughness of $2 \mu \mathrm{m}$, as specified by the manufacturer) with a diameter of $40 \mathrm{~mm}$ and a gap of $800 \mu \mathrm{m}$. A solvent trap was used to avoid any solvent evaporation and to assure a uniform temperature in the sample. The whole gelation process was tracked using the multiwave analysis tool of the TA Instruments Trios software, which consists in continuously applying a periodic shear deformation, as given in Eq. (1), obtained from the superposition of four different sinusoids ( $\mathrm{m}=4$ in Eq. (1)) with characteristic frequencies of 1, 5, 20 and 50 $\mathrm{rad} / \mathrm{s}$. The parameters of the specific deformation function are reported in Table 1 . The maximum value of the shear deformation is $3.84 \%$, which falls within the LVR for our gels 
(see SI-2). Given the sensitivity of stress-controlled instruments to inertia problems, a frequency sweep was performed on the sample with the lowest values of both the storage and the loss moduli and confirmed that the measurements were not affected by any inertia of the instrument (see SI-3). To obtain significant data, the minimum acquisition time for each single data point should be at least equal to one period of the fundamental frequency [22], which in this case is $6.28 \mathrm{~s}$. On the other hand, the acquisition time represents the accuracy of the gelling time evaluation; hence, the lower, the better. An acquisition time of $7 \mathrm{~s}$ showed to be reasonably lower than the characteristic gelation time for the majority of the experimental conditions investigated and therefore was chosen for all the experimental conditions. All the tests were performed for samples containing different mass fractions of glycerol in a range of temperatures from $25^{\circ} \mathrm{C}$ to $60^{\circ} \mathrm{C}$. All the concentrations and operating temperatures considered are summarized in the Supplementary Information, section SI-4. The samples, prepared as specified in Section 2.1, were loaded on the Peltier plate at room temperature. The final temperature for each test was set only after the loading process had ended. This procedure allows reducing any possible effect induced by the loading step on the tests reproducibility.

\subsection{UV-vis Spectroscopy}

The gelation process was also tracked using a UV-visible spectrophotometer (USB2000+, Ocean Optics) with Peltier-controlled Cuvette Holder (CUV-QPOD, Ocean Optics). The light absorbed by each sample $(1 \mathrm{ml})$ was measured every 0.5 seconds at the full range of wavelengths. The trend of the absorbance function with time was compared with the rheological measurements using a wavelength of $500 \mathrm{~nm}$, which is the standard employed for turbidity measurements [28-30]. The glycerol spectrum was used as reference for the absorbance calculations. All measurements were repeated for the same range of concentrations and temperatures specified in the previous section. The results obtained were 
used to confirm the mechanism proposed for the gelation process and will be presented in the last part of the results section.

\section{Results and Discussion}

\subsection{Characterization of the gelling transition}

The evolution of the viscoelastic properties follows the same trend in all conditions studied. An example is shown in Figure 2 where both the storage and loss moduli are presented for sample X4 (40\%wt glycerol/60\%wt Carbopol/PEG dispersion) at a gelation temperature of

$40{ }^{\circ} \mathrm{C}$. Three different stages can be clearly observed for both properties: a first stage in which both moduli remain constant or increase very slowly, a transition stage with a comparatively rapid monotonic increase of both $G^{\prime}$ and $G^{\prime \prime}$, and a final plateau region in which the moduli slowly increase, eventually reaching their equilibrium values. The trends of $G^{\prime}(t)$ and $G^{\prime \prime}(t)$ and their similar behaviour have already been observed for Carbopol dispersions in PEG400 by Bonacucina et al. [11] and can be explained by considering the final structure that this type of gel has. Carbopol is known to form a microgel structure, consisting of individual soft swollen particles which become packed above a critical concentration, being therefore confined by their neighbours [6,8,31]. During this process the viscous modulus increases as a consequence of the thickening of the whole solution, while the elastic component rises because of the increased interaction (bridging) between the swelling polymer particles, which eventually leads to complete jamming $[14,32]$. The bridging between Carbopol particles in the presence of protic solvents can be mainly ascribed to the formation of hydrogen bonds between solvent molecules and multiple Carbopol particles, which induces the formation of a network [33]. Generally, loss and storage moduli of Carbopol gels depend strongly on Carbopol concentration [9], crosslinking degree of the Carbopol used, solvent $[4,5,10,11]$ and shear history to which the initial dispersion is 
subjected during the gelation process [12,34]. In this specific case it is reasonable to state that the final plateau values of $G^{\prime}$ and $G^{\prime \prime}$ at a specific temperature in different samples predominantly depend on the sample composition. Since we tested only one type of Carbopol molecule, there is no dependence on the crosslinking degree. Additionally, any effect of the shear rate is negligible considering that the applied strain is small enough to avoid any disruption of the internal structure of the sample. The dependence on sample composition includes two different aspects: solvent composition and Carbopol concentration. The two variables cannot be completely decoupled considering that each sample has a different solvent composition and Carbopol concentration. However, in a previous study by Chu et al. [10], the authors pointed out that the final viscoelastic behaviour of Carbopol gels is not strongly affected by the composition of the solvents when using non-aqueous mixtures, while a major effect is observed with addition of water.

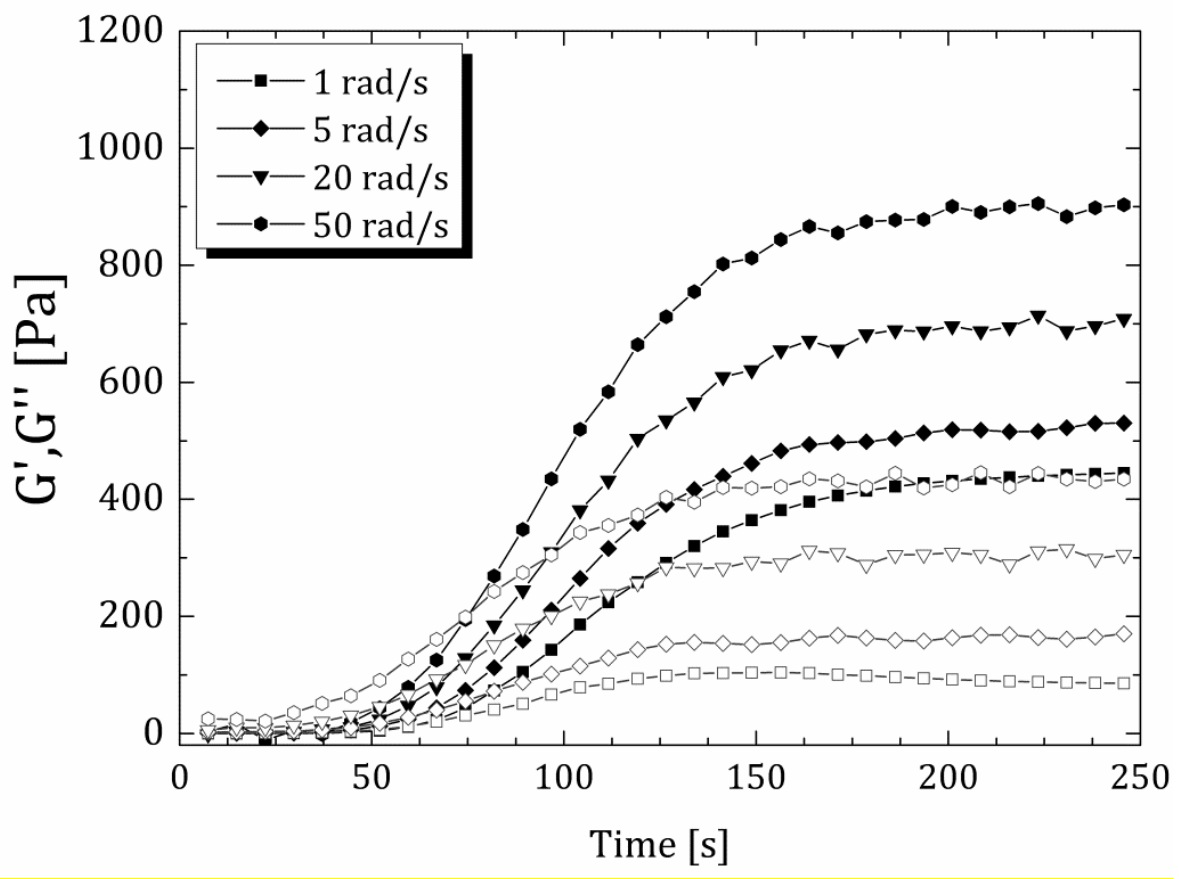

Figure 2 - Time evolution of the storage modulus (closed symbols) and loss modulus (open symbols) obtained at four different frequencies for a premixed sample of $40 \% \mathrm{wt}$ glycerol (X4) at the operating temperature of $40{ }^{\circ} \mathrm{C}$. 
Therefore, it is assumed that the final viscoelastic behaviour of our system is mainly dependent on Carbopol concentration. Figure 3 shows the plateau values extrapolated at each frequency for all samples reported in SI-4.1 for a gelation temperature of $40{ }^{\circ} \mathrm{C}$, with the error bars referring to the standard error obtained from three multi-wave test repetitions. As can be noticed, the experiments show a good reproducibility of the final state of the Carbopol dispersions. For Carbopol mass fractions higher than 1.2\%wt (samples X1-X6) the final activated matrix exhibits a weak gel behaviour, characterized by an almost constant elastic component that dominates the viscous one, which, however, is not negligible $[1,35,36]$. With an increase in Carbopol content, the elastic behaviour becomes stronger and the gap between $G^{\prime}$ and $G^{\prime \prime}$ widens. On the other hand, a further dilution of the original dispersion with Glycerol (samples X7 and X8) leads to a viscous liquid with comparable values of $G^{\prime}$ and $G^{\prime \prime}$. A clearer analysis of these results can be obtained by observing the trends of the loss tangent $\tan \delta$ with the actual mass fraction of Carbopol for all the temperatures studied at a representative frequency of $1 \mathrm{rad} / \mathrm{s}$ (Figure 4). The transition to a weak gel behaviour starts when the concentration of swollen Carbopol particles is enough to have significant interactions to form a micro-network in the solvent bulk. As specified above, in this event the elastic modulus prevails over the loss modulus. As a consequence, the loss tangent decreases to a value lower than 1 . With a further increase in Carbopol content, the system reaches a packed condition, with consequent plateauing of the viscoelastic properties. The mass concentration of Carbopol at which this condition is reached is referred to as overlapping concentration. As can be observed in Figure 4, for all temperatures the characteristic concentration at which the stable mixtures start to behave as a weak gel lies around $1.2 \%$ (sample X7), as highlighted from values of $\tan \delta$ lower than 1, while the overlapping concentration is around 2.8\% (sample X3). Indeed, for Carbopol concentrations higher than this value, the loss tangent remains constant at a minimum plateau value in the order of 
magnitude of 0.1 and it is weakly dependent on the oscillation frequency, which is typical of soft-glassy materials [37]'[38]. Given the different properties of the final matrices obtained at different Carbopol concentrations, the definition of a characteristic time of the process for all concentrations investigated is not straightforward. Winter and co-workers [19,21] were able to study the viscoelastic behaviour of crosslinking gels at the gel point, defining a criterion to determine a characteristic gel time based on the self-similarity found between the viscoelastic liquid in the pre-gel conditions and the viscoelastic solid in the post-gel condition. As a consequence of this, at the gel point, the material presents a power law dependence of the loss and storage moduli on the frequency:

$$
G^{\prime \prime}(\omega) \sim G^{\prime}(\omega) \sim \omega^{n}
$$

Therefore, the loss tangent is frequency-independent and the gel point can be obtained as the time at which the loss tangent crosses for all oscillation frequencies $\omega$. 

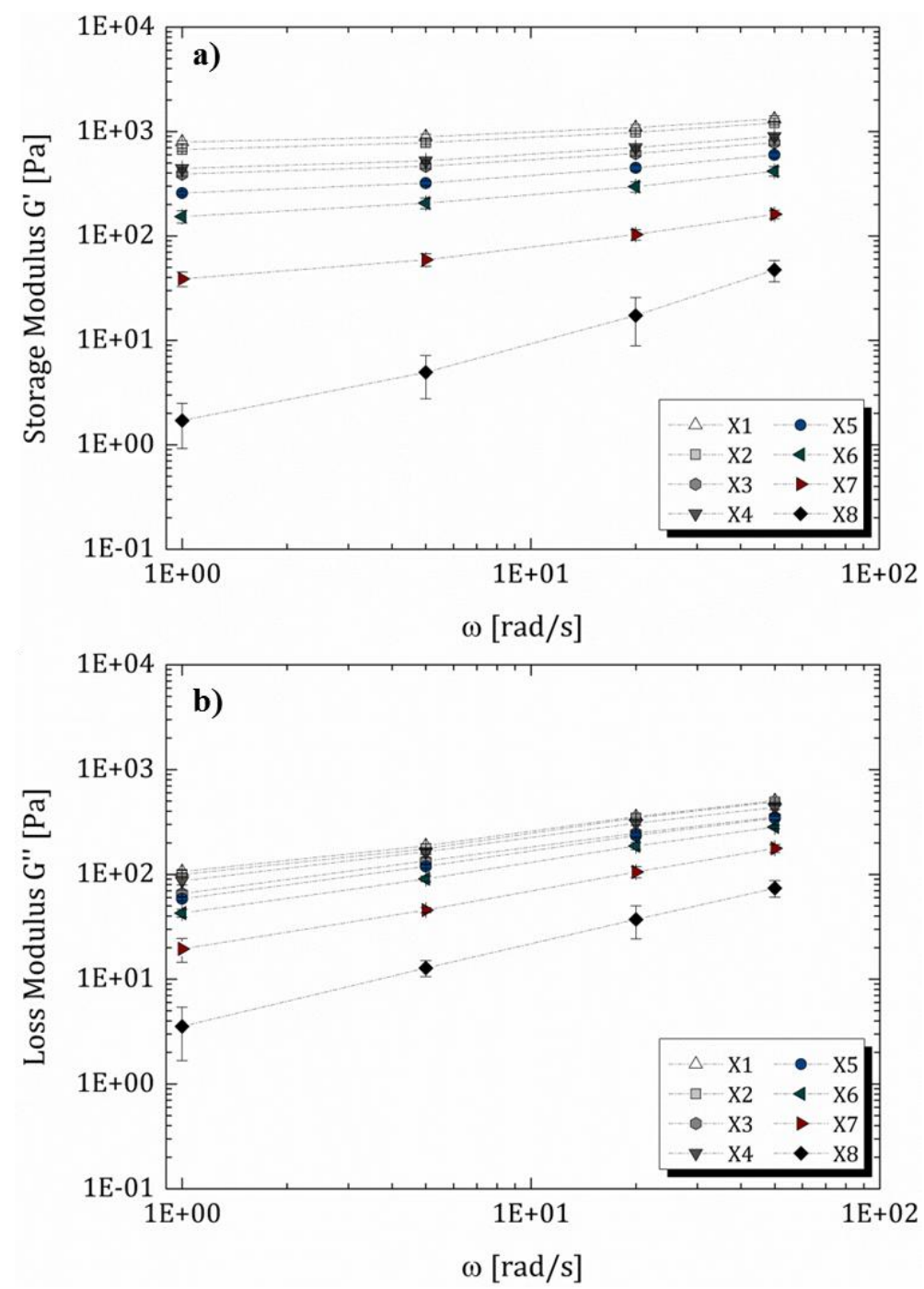

Figure 3 - a) Storage and b) Loss modulus plateau values at the four frequencies tested for all samples at $40^{\circ} \mathrm{C}$.

Even though this criterion was developed for cross-linking gels [21,39], the power law dependence of the viscoelastic properties at the sol-gel transition has been found to hold for different kinds of gelling systems, which present a percolated structure [24,40-42]. In Figure 5 we report a representative example of the time evolution of the loss tangent for all frequencies studied with the aim of highlighting possible differences in the transition that the dispersions experience when the sample composition is varied. Specifically, the three graphs show the results obtained for three samples containing an increasing mass fraction of Carbopol at $40{ }^{\circ} \mathrm{C}$. As expected, for samples with the lowest concentration of Carbopol (sample X8) the mixture does not present a true cross-over, the loss tangent being higher at 
lower oscillation frequencies, which is typical of viscoelastic liquids [39]. A neat cross-over is observed for sample $\mathrm{X} 4$, which confirms the presence of a percolated structure for intermediate Carbopol concentrations. Finally, for the most concentrated samples (sample $\mathrm{X} 2$ ), the cross-over is again not present and the loss tangent is predominantly higher at higher frequencies, which is typical of viscoelastic solids, thus possibly indicating that the Carbopol volume fraction in the sample is high enough to reach a complete jammed configuration.

Despite the possible applicability of the method to this specific case, the criterion gives an indication of the gelation time and not a continuous description of the time evolution of the material independently of its final state, which is fundamental from a processing viewpoint and our main goal. Therefore, a kinetic analysis was performed on the evolution of the storage modulus $G^{\prime}(t)$, which is directly related to the build-up of internal micro-structures $[32,43]$. Hence, to obtain a continuous description of the evolution of the material, we defined a structural "degree of conversion" $X_{S}$ as a normalisation of the storage modulus:

$$
X_{S}\left(t, \omega_{i}\right) \equiv \frac{G^{\prime}\left(t, \omega_{i}\right)-G_{0}^{\prime}\left(\omega_{i}\right)}{G_{\infty}^{\prime}\left(\omega_{i}\right)-G_{0}^{\prime}\left(\omega_{i}\right)}
$$

where $G^{\prime}\left(t, \omega_{i}\right)$ is the storage modulus at time $t$ and frequency $\omega_{i}, G^{\prime}{ }_{0}\left(\omega_{i}\right)$ is the plateau of the storage modulus for the oscillation frequency $\omega_{i}$ at the initial stages of the gelation process and $G^{\prime}{ }_{\infty}\left(\omega_{i}\right)$ is the final equilibrium plateau value. 


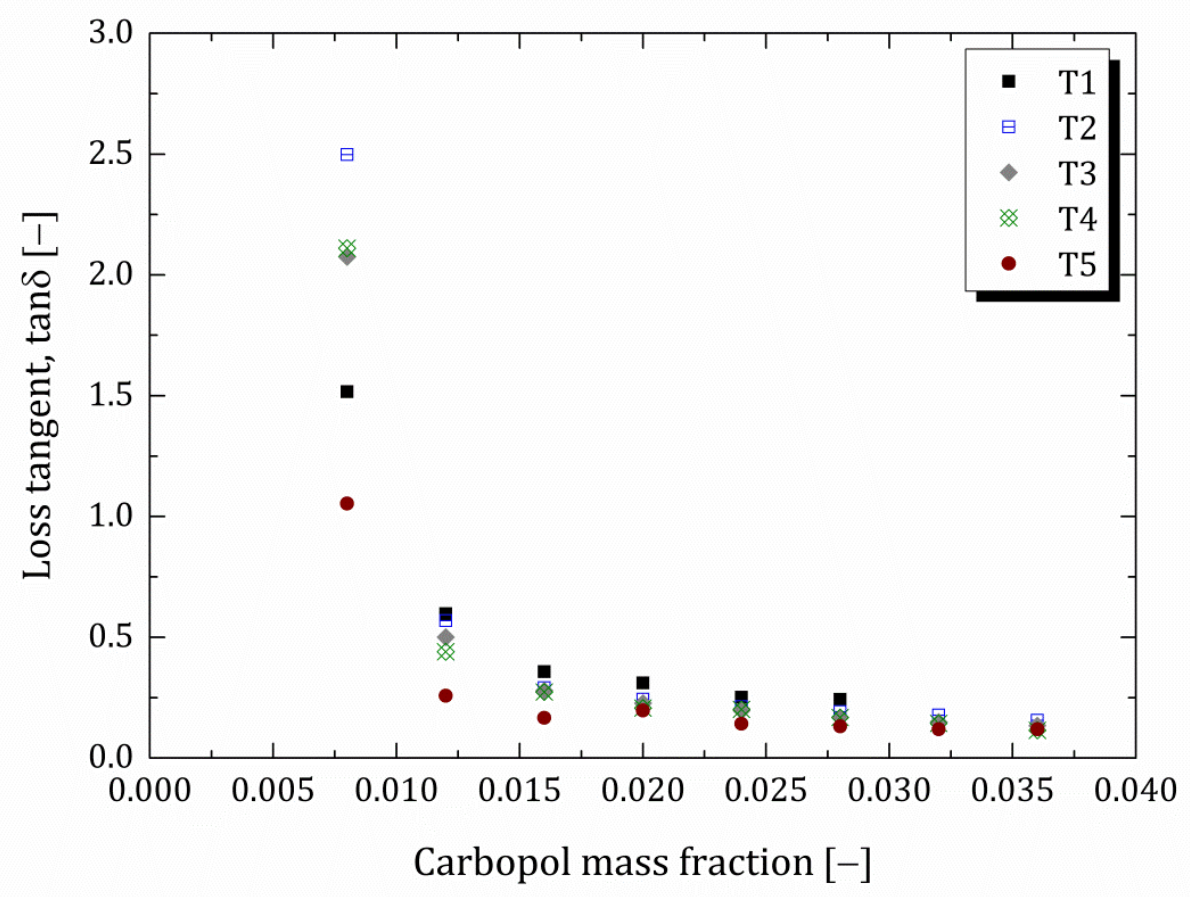

Figure 4 - Loss tangent against effective Carbopol mass fractions reported for all the temperatures investigated for a representative oscillation frequency of $1 \mathrm{rad} / \mathrm{s}$.

The mathematical description of the time evolution of $X_{S}$ can be used to derive a generalized kinetic model for the complete uncoiling and swelling of the Carbopol molecules with direct insight in the time evolution of their structures. The shape of $X_{S}$ maintains the same characteristic of the curves observed in Figure 2, but thanks to the normalisation, the curves at different frequencies overlap, thus allowing a unique fitting for all the samples at a specific operating temperature (Figure 6). 

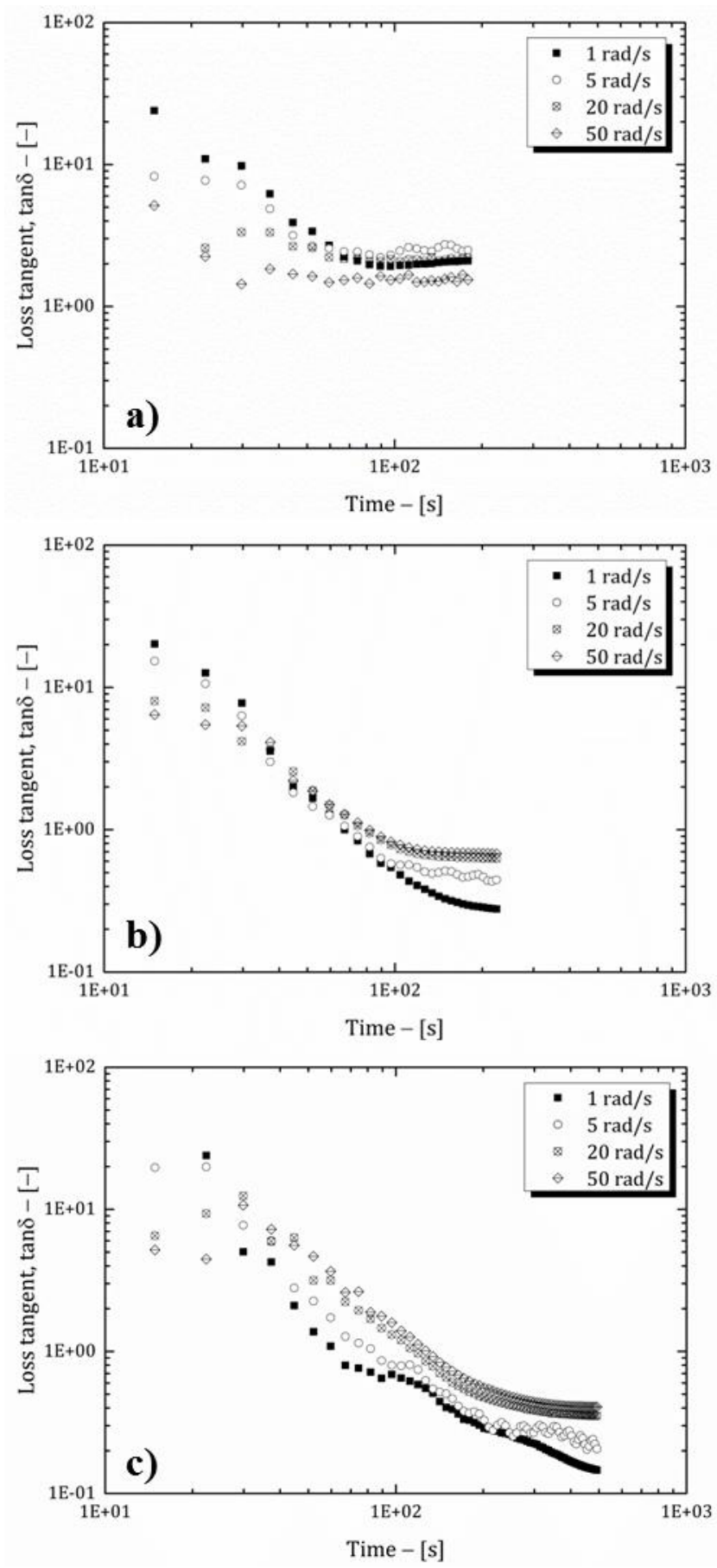

Figure 5 - Time evolution of the loss at the four frequencies tested in the multi-wave analysis obtained at $40{ }^{\circ} \mathrm{C}$ for: a) $20 \%$ wt Carbopol-PEG400 dispersion/80\%wt glycerol (X2); b) 40\%wt Carbopol-PEG400 dispersion/60\%wt glycerol (X6); c) 80\%wt CarbopolPEG400 dispersion/20\%wt glycerol (X2). 


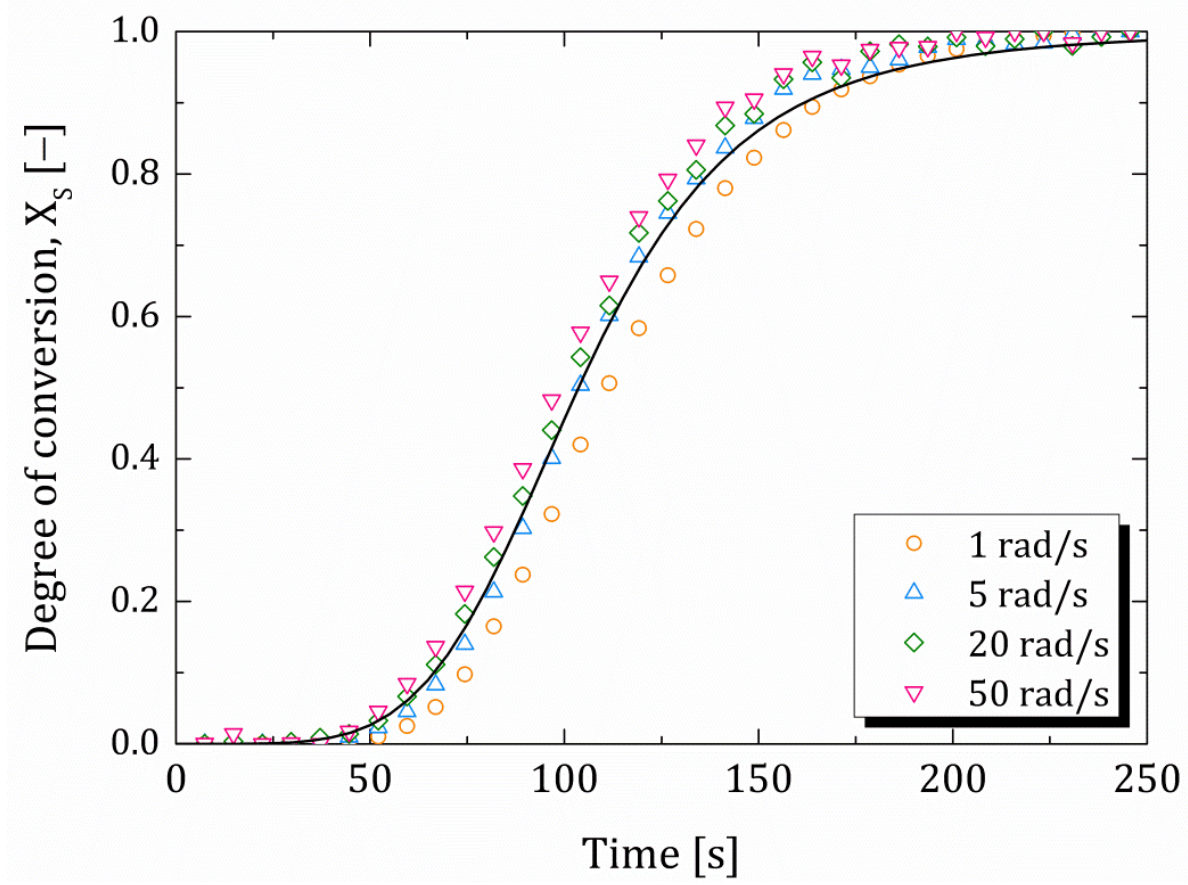

Figure 6 - Structural degree of conversion $X_{S}$ obtained at four different frequencies for a premixed sample of $40 \%$ wt glycerol (X4) at the operating temperature of $40{ }^{\circ} \mathrm{C}$. The solid line reports the fitting applied as described in Eq. (6).

As can be seen $X_{S}$ does not significantly depend on the oscillation frequency which allows us to express its time evolution through a modified version of the Hill equation [43,44], applied to all samples:

$$
X_{S}(t)=\frac{t^{n}}{t^{n}+\theta^{n}}
$$

where $n$ and $\theta$ are the two parameters of the kinetic function: $\theta$ is the gelation half-time, defined as the time at which the system reaches half of its conversion [43], while $\mathrm{n}$ is correlated to the slope of the function $X_{S}(t)$ at the gelation half-time. The value of this slope, denoted as $P$, is given by the following relation (for further details see $\mathbf{S I - 5}$ ):

$$
\left.P \equiv \frac{d X_{S}}{d t}\right|_{t=\theta}=\frac{n}{4 \theta}
$$

$P$ can be physically interpreted as the characteristic rate of the dissolution process. Both parameters $(\theta$ and $P)$ are used to depict the kinetic behaviour of the process upon addition of glycerol. It should be noted that the actual concentration of Carbopol present in each tested 
sample changes when the second solvent is added. Naturally, the polymer concentration affects the rate of the process; however, our interest was to study gelation when different fractions of the two main liquid streams, i.e. the polymeric dispersion (PEG400/Carbopol, with fixed Carbopol concentration), treated as a whole, and the glycerol, are mixed together in a process. Therefore, in the following analysis all parameters are directly referred to the glycerol content of the samples studied.

\subsection{Influence of glycerol dilution}

The effect of the addition of glycerol on the gelation process can be qualitatively observed in Figure 7, where the time evolution of the degree of conversion $X_{S}$ is plotted for four different samples at a fixed temperature of $40{ }^{\circ} \mathrm{C}$. The rate of conversion clearly increases with the glycerol mass fraction. This trend can be quantified by plotting the kinetic parameters $\theta$ (gelation half-time) and $P$ (transition rate) characterizing the function $X_{S}(t)$ (see Eqs. (6) and (7)) against the mass fraction of glycerol, as shown in Figure 8 and Figure 9, respectively, for the five temperatures tested. It is clear that the whole process is dramatically affected by the mass fraction of glycerol present in the sample; for all temperatures, an increase in glycerol concentration results in a decrease of the gelation half-time $\theta$ as well as an increase of the rate of transition $P$. This effect is most probably caused by the better affinity that glycerol has as a solvent for Carbopol molecules compared to PEG400. Given the crosslinked

nature of Carbopols, the uncoiling process in the cases considered does not implicate a disruption of the polymer molecules but an uncoiling of their collapsed dry structure with consequent swelling. The whole mechanism depends on the affinity with the surrounding molecules of solvent and the solvent diffusion coefficient [45]. 


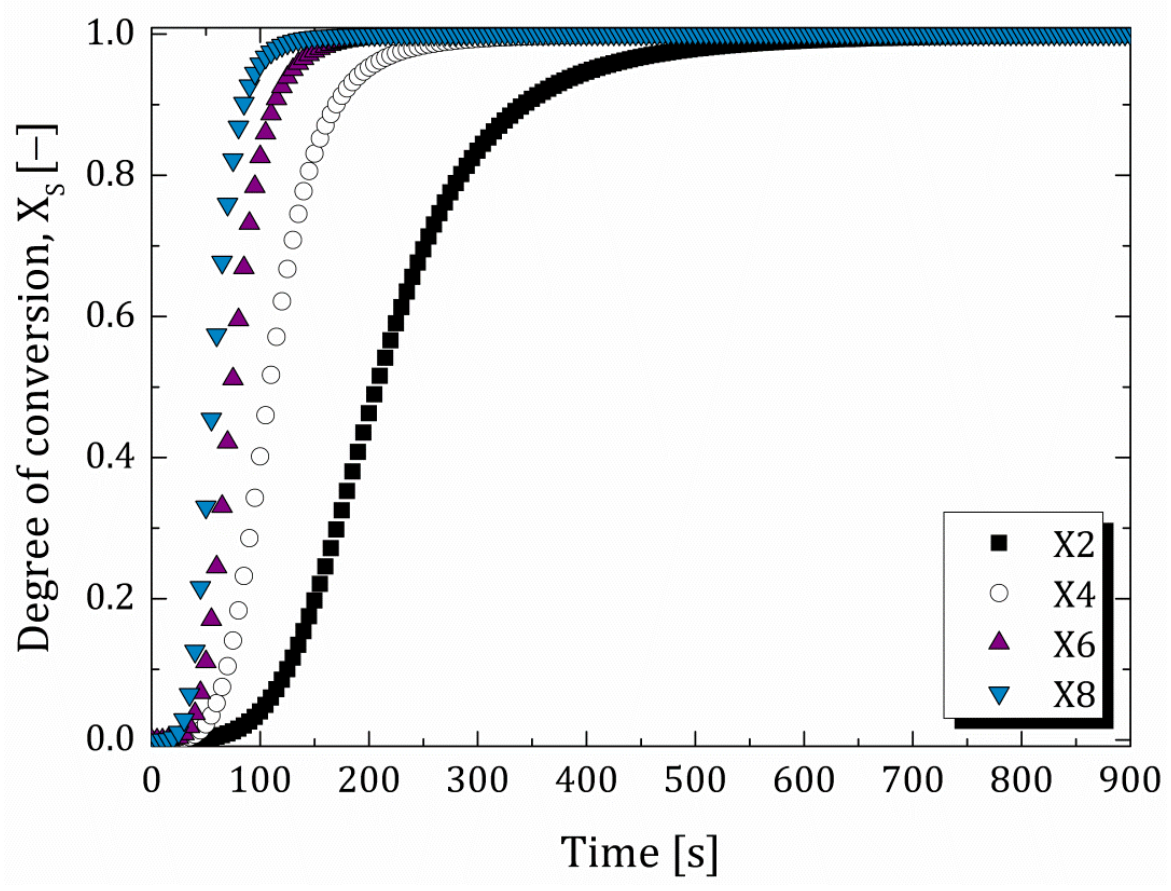

Figure 7 - Time evolution of the structural degree of conversion $X_{S}$ obtained at a gelation temperature of $40^{\circ} \mathrm{C}$ for four distinct samples each containing a different amount of glycerol (20\%wt, 40\%wt, 60\%wt and 80\%wt of glycerol, following the legend order).

Following the theory developed by Hansen [45], the polymer solubility parameters depend upon three interactions: (i) a dispersive interaction, which is the attractive force present in all molecules caused by the electromagnetic field induced by the electrons motion around the atom nucleus, (ii) a polar cohesive force, caused by permanent dipole-dipole interactions, and finally (iii) a molecular interaction caused by hydrogen bonding, which is usually more relevant in polymer dissolution than the previous two. Hence, from the observation of the chemical structure of the two solvents used, glycerol presents a higher density of hydroxyl groups than polyethylene glycol, which gives the molecule a higher polarity and a better ability to form hydrogen bonds with the lateral carboxyl groups of the acrylic acid; it is thus thermodynamically preferred. Furthermore, glycerol is smaller than PEG400 and can more easily penetrate the crosslinked network of Carbopol. 


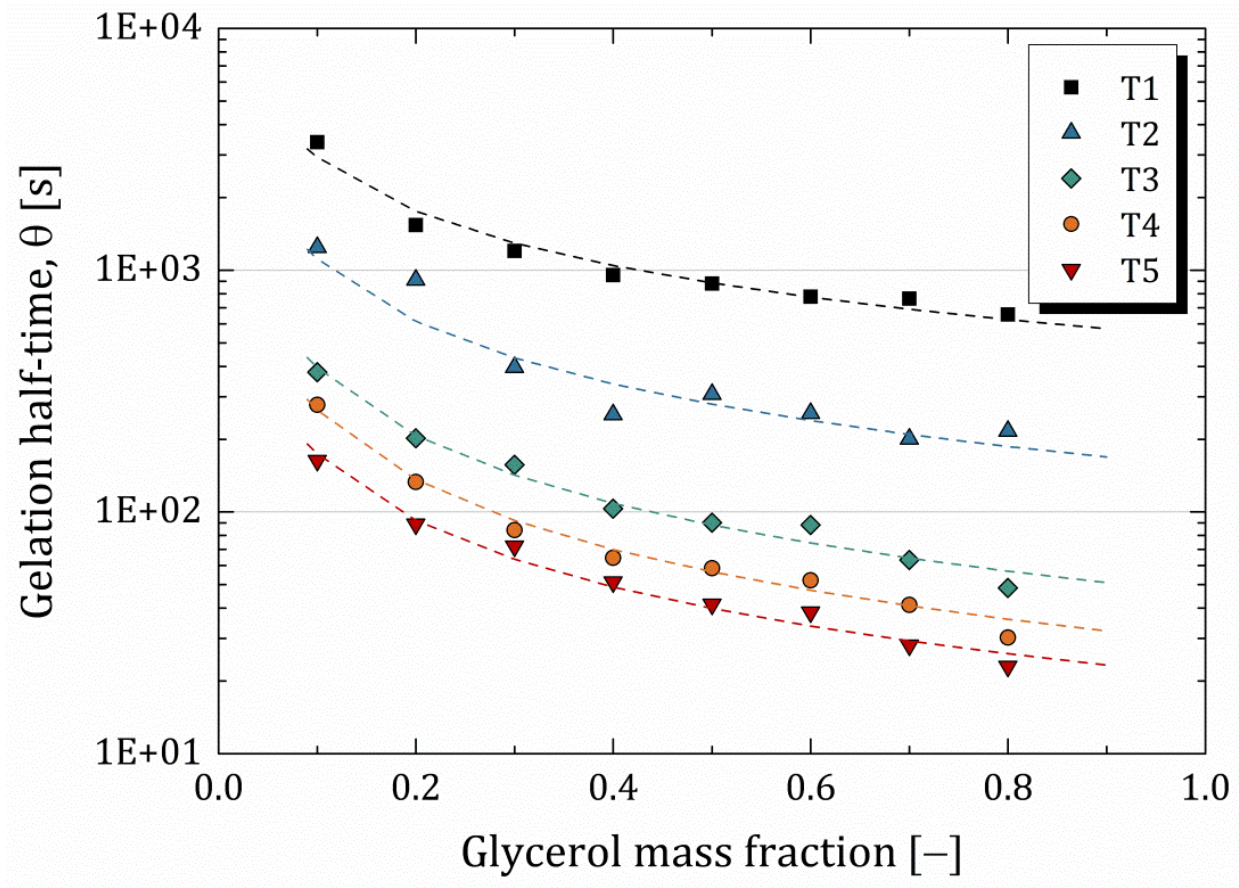

Figure 8 - Gelation half-time $\theta$ against the glycerol mass fraction in the samples at the five temperatures tested $\left(25,30,40,50\right.$ and $60{ }^{\circ} \mathrm{C}$, following the legend order). The symbols represent the values obtained from the fitting of the structural conversion degree (Eq. (6)); the dashed lines are the power law fittings at each temperature, obtained from Eq. (8).

In considering the effect of the glycerol content on the process kinetics, it should be taken into account that the corresponding amount of dispersed polymer in each sample decreases upon glycerol addition, as indicated by the real mass fractions in SI-4.1. On the assumption that in the initial conditions, when the powder is uniformly dispersed in the liquid bulk, each Carbopol particle uncoils and swells independently of the others, the lower the number of swelling particles is, the longer the time needed to reach complete packing conditions in a confined liquid volume should be. A schematic of this assumption is shown in Figure 10, where Carbopol particles are idealized as perfect spheres. In the presence of a uniform liquid solvent, particles swell at the same rate regardless of their initial concentration. At time $t_{2}$ (third column) the sample with the higher number of particles reaches the packing condition; after this point the particles start to deform as a result of the contact with their neighbours. The particles in the lower concentration sample, on the other hand, continue to swell until 
packing is reached. Therefore, when glycerol is added to the polymeric dispersion there are two counteracting effects that influence the process kinetics, (i) the dilution which tends to decrease the gelation rate and (ii) the higher percentage of glycerol, which tends to increase the process kinetics. From experimental observations, the second one seems to dominate. To verify the hypothesis on the effect of dilution, we applied the same time-resolved rheometry technique to different samples containing PEG400 (but no glycerol) with increasing concentrations of Carbopol, i.e. $0.5 \%$ wt, $2 \%$ wt and $6 \%$ wt at $40{ }^{\circ} \mathrm{C}$. As shown in Figure 11, the higher the Carbopol concentration is, the faster the storage modulus increases, as predicted.

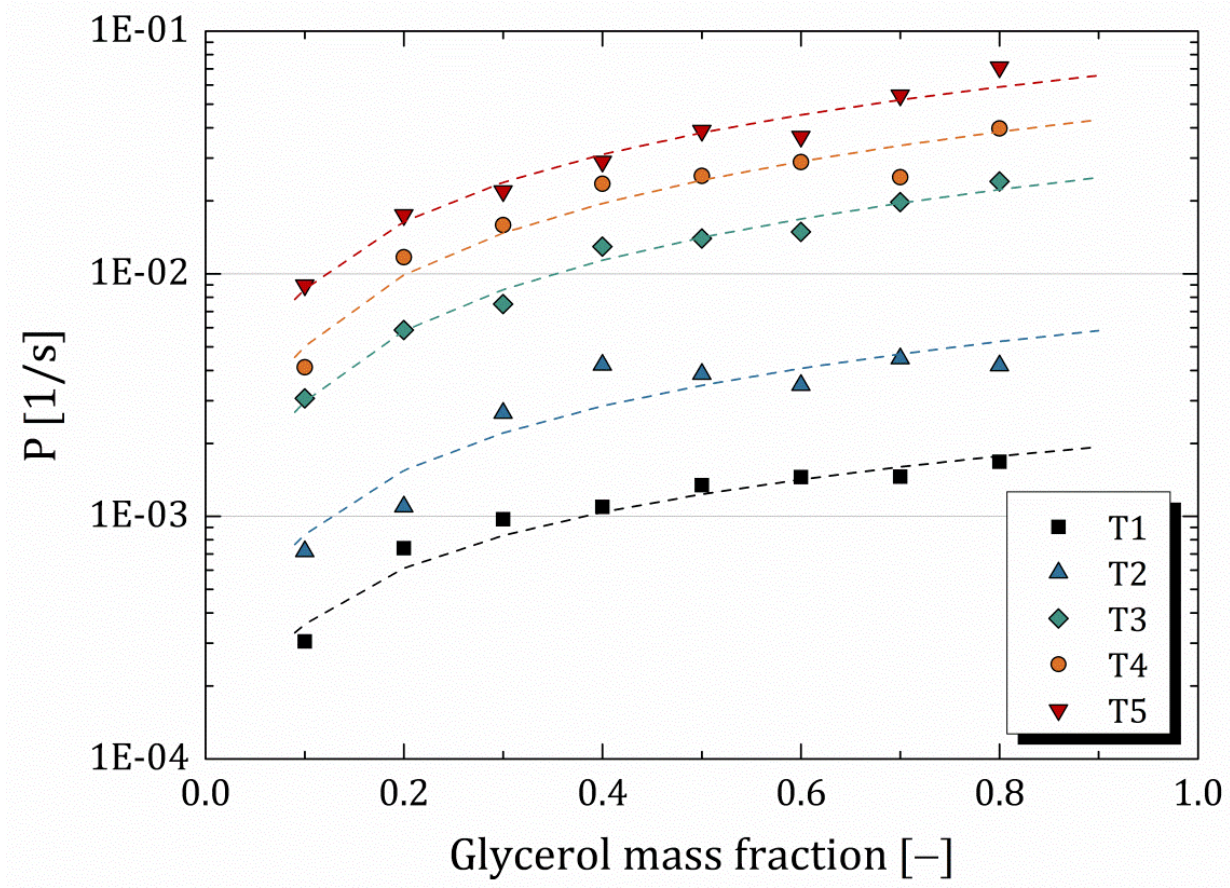

Figure 9 - Transition rate $P$ against the glycerol mass fraction present in the samples at the five temperatures tested $\left(25,30,40,50\right.$ and $60^{\circ} \mathrm{C}$, following the legend order). The symbols represent the values obtained from the fitting of the structural conversion degree (Eq. (6)); the dashed lines are the power law fittings at each temperature obtained from Eq. (9).

This trend can be clearly observed comparing the results obtained at the two higher concentrations (2\% wt and 6\% wt in Carbopol), where in the first case there is only a slight increase of $G^{\prime}$ over time, while in the second the increasing monotonic trend is evident. 
However, even at the highest mass fraction of Carbopol $(6 \% \mathrm{wt})$, which is higher than the maximum Carbopol mass fraction considered in the samples of interest $(3.6 \% \mathrm{wt}$ in sample $\mathrm{X} 1$ ), the storage modulus is far from reaching a plateau value, in opposition with what it can be directly observed for the same operating condition for sample X1, reported in Figure 11. Therefore, using only PEG400, the process is slower than what was observed in the presence of glycerol, thus confirming its dominant effect.

\section{$t_{0}$}
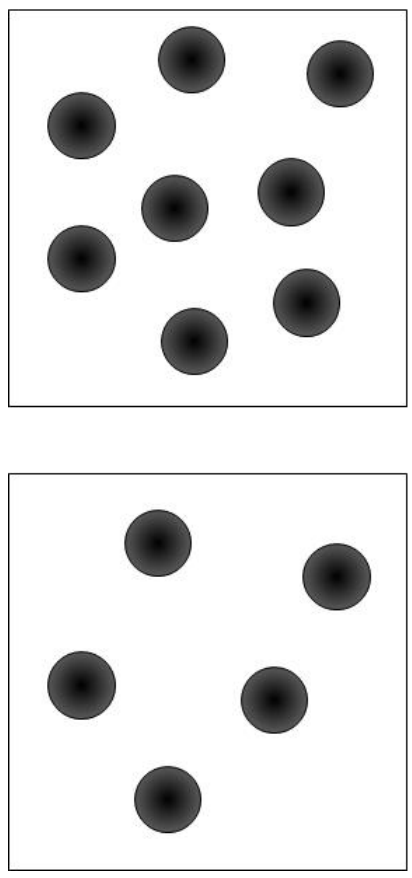

$t_{1}>t_{0}$
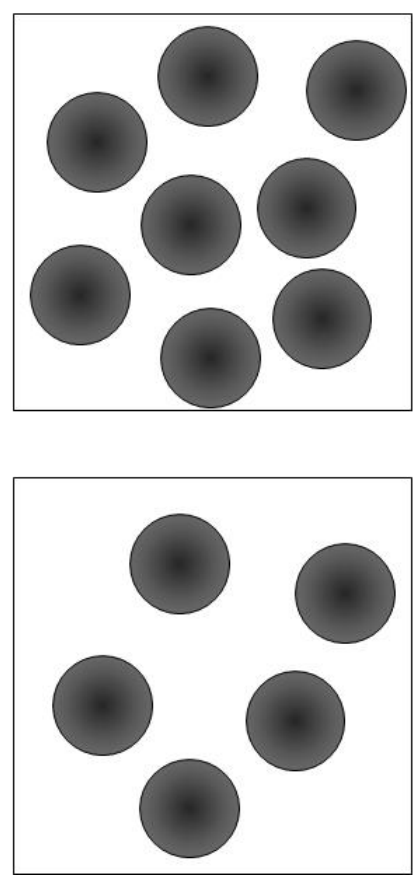

$t_{2}>t_{1}$
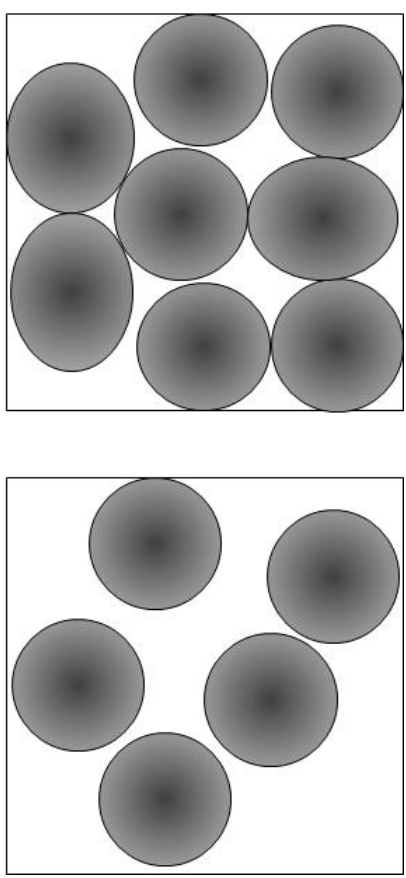

Figure 10 - Simplified schematic of the swelling behaviour of Carbopol particles dispersed in a single solvent at two different polymer concentrations (top row: high concentration; bottom row: low concentration). At the initial time, $t_{0}$, each particle starts to uptake solvent and consequently swells independently from its neighbours $\left(t=t_{1}\right)$ until reaching the packing condition. At time $t_{2}$ the high concentration sample has already reached the final packing condition because of the higher number of particles present. The low concentration sample can still potentially swell until reaching the final packed condition. 


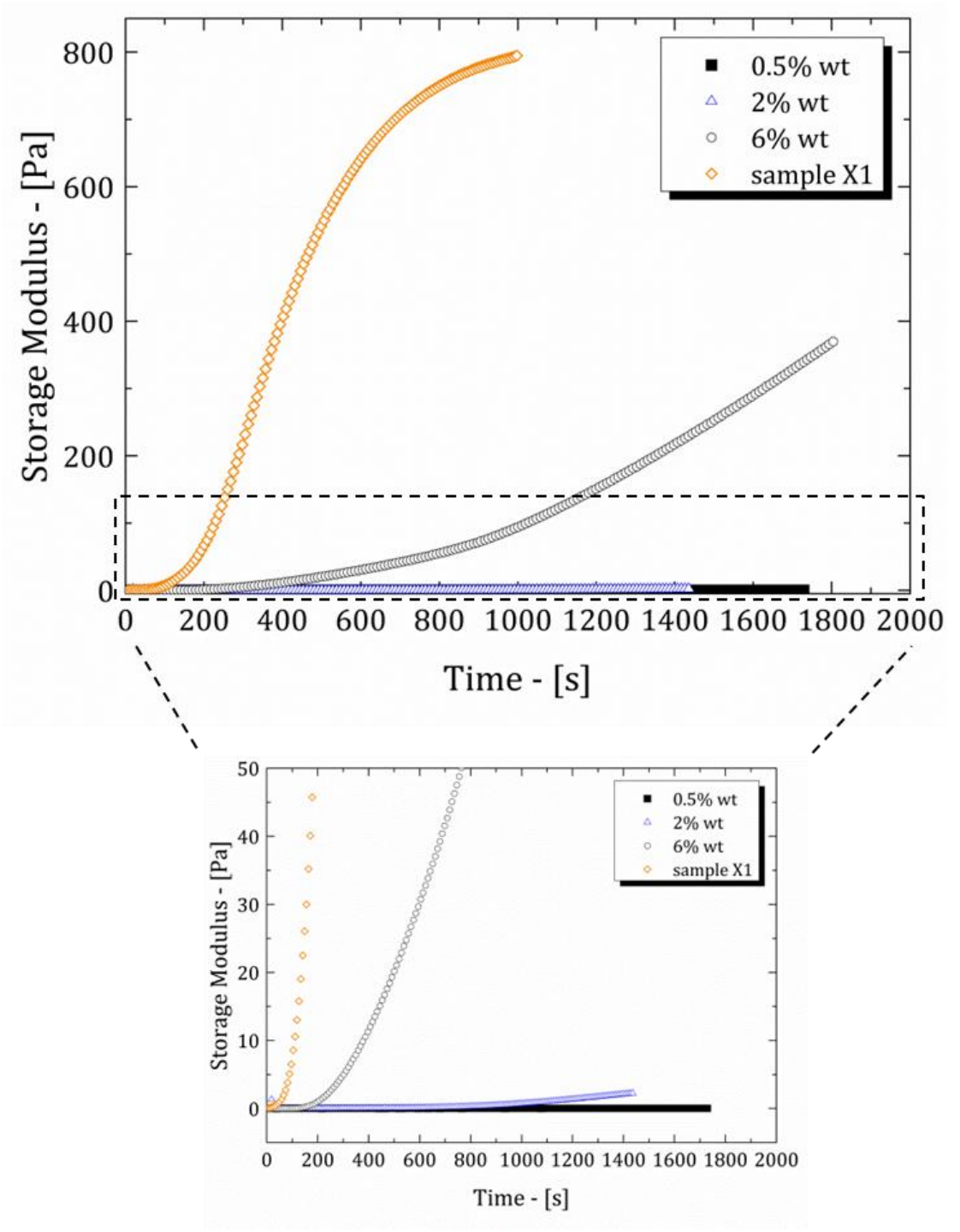

Figure 11 - Time evolution of the storage modulus for three different concentrations of Carbopol dispersed in PEG400 at $40^{\circ} \mathrm{C}$ and sample X1(containing 3.6\%wt of Carbopol). The FTMS test was performed in the same conditions as the main samples but, for clarity, only the curves obtained at an oscillation frequency of $1 \mathrm{rad} / \mathrm{s}$ are reported.

\subsection{Influence of operating temperature}

The operating temperature exhibits a similar effect to that of glycerol concentration on the process kinetics, as can be observed in Figure 12, where the structural conversion degree is plotted against time for sample X4 for the five temperatures studied. The higher the operating temperature is, the faster the gelation progresses. This trend can also be observed in Figure 9, where an increase in temperature shifts the characteristic transition velocity to higher values. 
For each temperature, a power law can be fitted to both parameters $\theta$ and $P$, obtaining the following equations:

$$
\begin{aligned}
& \theta=k_{\theta}\left(x_{g l y}\right)^{-m_{\theta}} \\
& P=k_{P}\left(x_{g l y}\right)^{m_{P}}
\end{aligned}
$$

The values of the fitting parameters, $k_{\theta}, k_{P}, m_{\theta}$ and $m_{P}$ are reported in Table 2. As expected, the constants $k_{\theta}$ and $k_{P}$ decrease and increase with temperature, respectively. On the other hand, the exponents $m_{\theta}$ and $m_{P}$ slightly decrease at $30{ }^{\circ} \mathrm{C}$ and $25^{\circ} \mathrm{C}$, respectively, whilst remaining roughly constant at the highest temperatures, with a mean value of around 0.956.

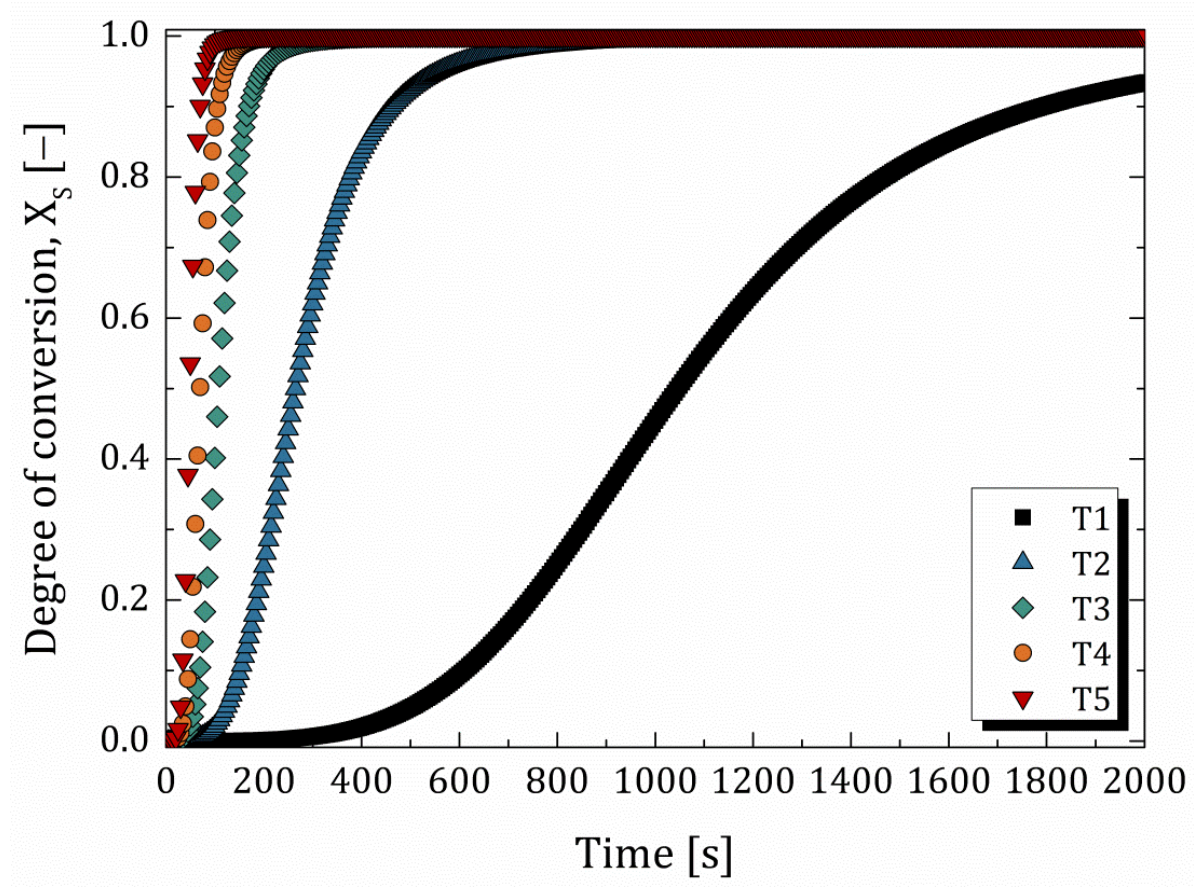

Figure 12 - Time evolution of the structural degree of conversion $X_{S}$ obtained at the five gelation temperatures tested $\left(25,30,40,50\right.$ and $60^{\circ} \mathrm{C}$, following the legend order).for sample X4 (40\%wt of glycerol content). 


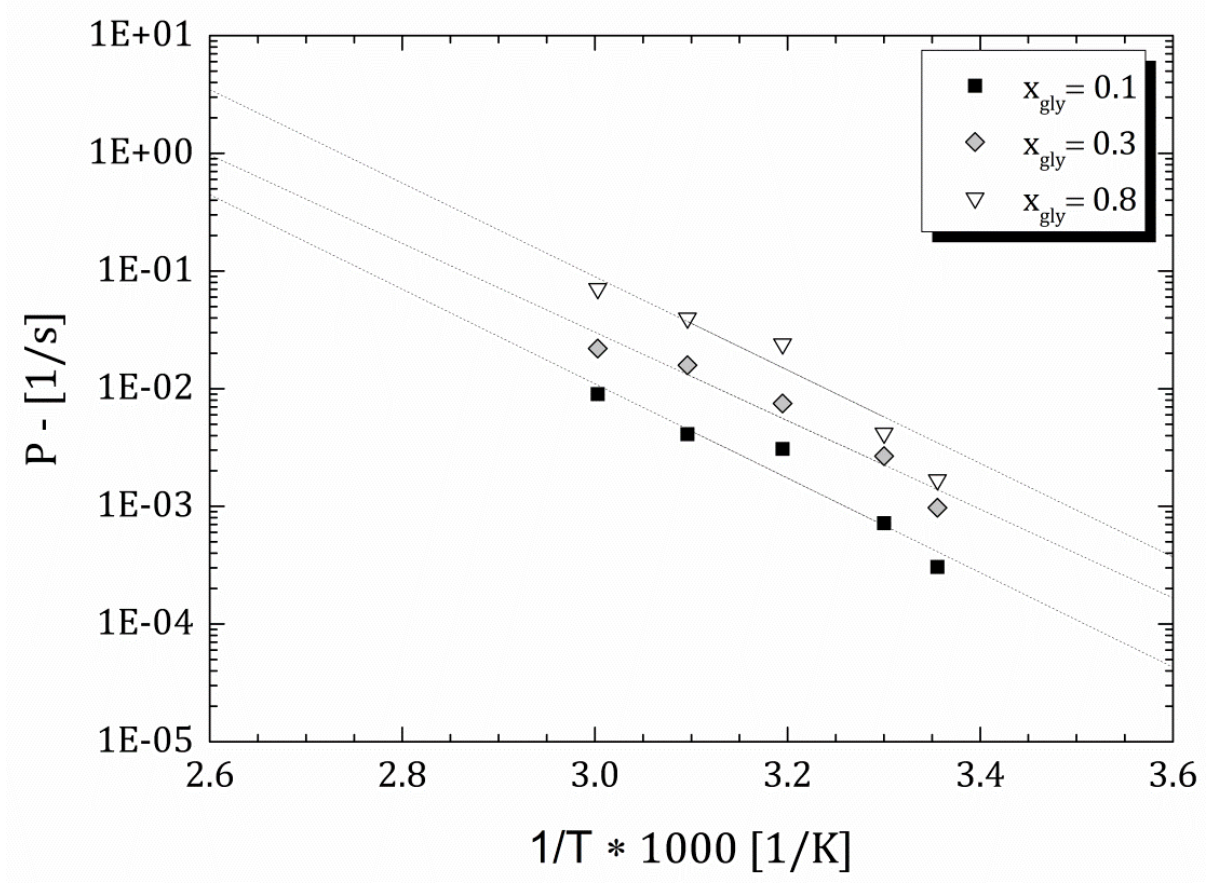

Figure 13 - Arrhenius plot of the transition rate P. For clarity only the values for three different samples are reported. The dashed lines are the corresponding fittings of the Arrhenius law.

The temperature dependence can be better understood by observing the Arrhenius plot.

Figure 13 shows the characteristic rate of the process $P$ against $1 / T$ for three different samples. A classic Arrhenius model can be fitted to all concentrations:

$$
P=K \exp \left(-\frac{\Delta E_{a}}{R T}\right)
$$

where $K$ is the pre-exponential constant, $\Delta E_{a}$ is the activation energy and $R$ is the ideal gas constant; the ratio $\frac{\Delta E_{a}}{R}$ can be further defined as an activation temperature $T^{*}$ [46]. Equation (10) can be written equivalently as follows:

$$
\ln P=\ln K-\frac{T^{*}}{T}
$$

The fitting parameters of this equation are reported in Table 3. For all samples the activation temperature appears to be reasonably constant; the average value together with the coefficient of variance $(\mathrm{CoV})$ is reported in Table 4. However, to formulate a coherent interpretation of 
this findings it is essential to get a clearer understanding of the mechanism of the whole gelation process. Therefore, in the following section a mechanism based on the theory of polymer dissolution is proposed.

Table 2 - Fitting parameters for the power laws defined in Eq. (8) and (9) obtained at each tested temperature.

\begin{tabular}{ccccc}
\hline $\mathbf{T}\left({ }^{\circ} \mathbf{C}\right)$ & $\boldsymbol{k}_{\boldsymbol{\theta}}(\mathbf{s})$ & $\boldsymbol{m}_{\boldsymbol{\theta}}(-)$ & $\boldsymbol{k}_{\boldsymbol{P}}(\mathbf{1} / \mathbf{s})$ & $\boldsymbol{m}_{\boldsymbol{P}}(-)$ \\
\hline \hline $\mathbf{2 5}$ & 529 & 0.743 & 0.002 & 0.767 \\
$\mathbf{3 0}$ & 154 & 0.859 & 0.006 & 0.882 \\
$\mathbf{4 0}$ & 46 & 0.932 & 0.027 & 0.967 \\
$\mathbf{5 0}$ & 29 & 0.958 & 0.048 & 0.979 \\
$\mathbf{6 0}$ & 21 & 0.915 & 0.072 & 0.923 \\
\hline
\end{tabular}

Table 3 - Linearized pre-exponential parameter and activation temperature obtained from the Arrhenius fitting of the transition rate P for all concentration tested.

\begin{tabular}{ccc}
\hline SAMPLE & Ln K (1/s) & $\boldsymbol{T}^{*}(\mathbf{K})$ \\
\hline \hline X1 & 23 & 9247 \\
X2 & 23.5 & 9094 \\
X3 & 22.5 & 8673 \\
X4 & 23.5 & 8878 \\
X5 & 25 & 9316 \\
X6 & 25 & 9323 \\
$\mathbf{X 7}$ & 26 & 9675 \\
$\mathbf{X 8}$ & 25 & 9146 \\
\hline
\end{tabular}

Table 4 - Mean value of the Arrhenius law parameters displayed in Table 5 and corresponding covariance, expressed as the ratio between the standard deviation of the parameter and its mean.

\begin{tabular}{ccc}
\hline & Mean value & $\operatorname{CoV}(\%)$ \\
\hline \hline $\boldsymbol{T}^{*}(\mathbf{K})$ & 9169 & 3.3 \\
\hline
\end{tabular}




\subsection{Mechanism for uncoiling/swelling of a Carbopol particle}

The dissolution process of a non-crosslinked polymer into a solvent involves typically three main steps: (i) solvent diffusion into the polymer matrix, (ii) formation of an external swollen gel-like layer (plasticization) and (iii) chain disentanglement with consequent transport of polymer chains into the solvent bulk [45,47]. Following these steps, at first the solvent penetrates into the polymer, creating an external swollen layer of polymer which expands in the opposite direction to that of the solvent flux. The swelling of the gel layer in the direction of the solvent bulk is counterbalanced by the polymer dissociation at the boundary between the gel layer and the solvent with consequent diffusion of polymer chains into the solvent bulk, which finally causes the complete dissolution of the initial polymer matrix [45]. All existing mathematical models are based on this qualitative description and mainly differ in the complexity with which the transport phenomena are described. A full classification of models available for dissolution of amorphous and semi-crystalline polymers can be found in the literature $[45,47]$. In case of a crosslinked system, as Carbopol molecules, the main difference with the description above is that single polymer chains do not dissolve in the solvent, as a result of the disentanglement of the polymer network, but the whole matrix uncoils and swells until reaching a final equilibrium condition, which depends on the type of solvent, temperature, polymer concentration and operating conditions [9]. Regardless of the different considerations used to model the various steps of a polymer dissolution process, the vast majority of the approaches developed over the years use a simple Fickian equation to describe the solvent transport inside the polymer bulk and consider the swelling process as a direct consequence of this diffusive flux $[45,48,49]$. Hence, in the absence of any polymer chains dissociation, with consequent diffusion in the solvent bulk, the characteristic time of the process is given by the time needed by the solvent to diffuse into the whole polymer network. Despite the higher complexity of the real system, this description can be directly 
applied to a single Carbopol particle, idealized as a spherical particle surrounded by the solvent (Figure 14). Under the hypothesis of a solvent diffusion-controlled condition, the solvent molecules penetrate into the crosslinked surface of the polymer particle passing through a superficial diffusion layer. During the diffusion process, the coiled Carbopol network starts to uncoil creating a swollen diffusion layer. On the external boundary (the one in contact with the solvent) this diffusion layer has a polymer equilibrium volume fraction $c_{E}$, which depends on the maximum chain stretching allowed at equilibrium, while on the internal boundary (the one in contact with the polymer network) it has a saturation equilibrium volume fraction $c_{S}$ [48], which depends on the specific polymer/solvent system. The diffusion layer proceeds in the direction of the polymer core as a reaction front, leaving behind the swollen Carbopol external layer, at a polymer volume fraction $c_{E}$. Following this description, in quiescent conditions the swelling of each Carbopol particle depends mainly on the initial size of the particle and on the solvent diffusion coefficient. A change in composition of the solvent affects significantly the value of the diffusion coefficient. For binary mixtures of solvents, it has been shown that the overall kinetics of solvent diffusion into the polymer network increases with an increase in the fraction of solvent with smaller molecular size [50]. Therefore, a higher fraction of glycerol is expected to have a positive effect on the process kinetics, as experimentally observed in this work. All these considerations can be qualitatively applied to the whole bulk of the studied system, showing that, in all conditions studied, the kinetics of the process is dominated by diffusion. 


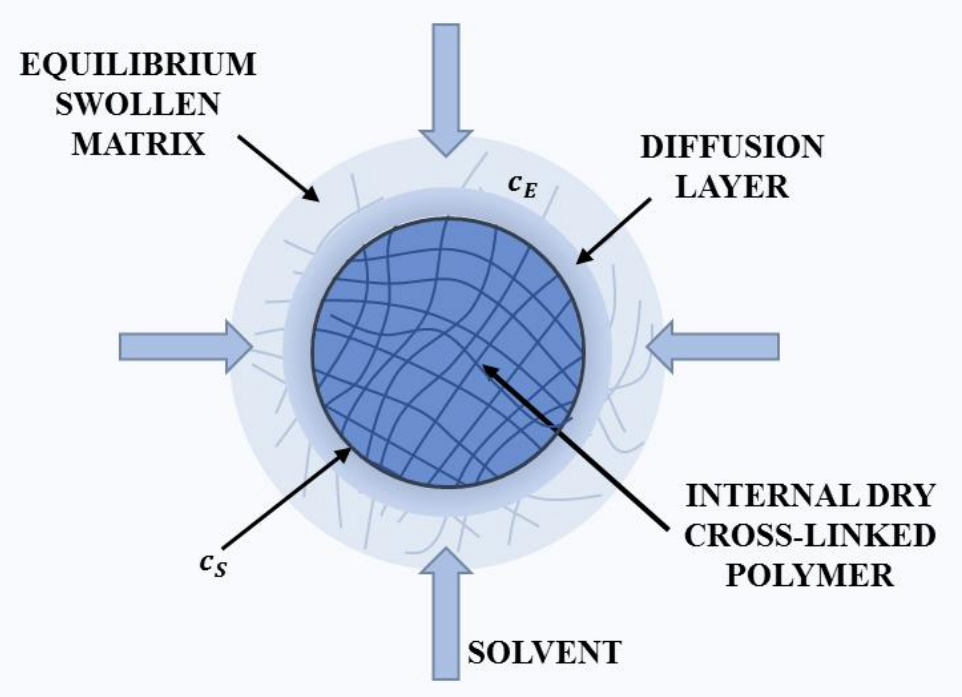

Figure 14 - Schematic of the mechanism of dissolution/swelling of a single Carbopol particle in a liquid solvent (thick arrows show solvent flux).

To confirm the dominance of the diffusive phenomenon in the mechanism of uncoiling/swelling of the Carbopol molecules, we tracked the time evolution of the UV-vis absorbance for the same conditions studied in the rheological analysis. At the wavelength considered $(500 \mathrm{~nm})$, only the dry core of Carbopol particles causes the absorption of the emitted light, whereas the swollen layers have a negligible absorption signal. Figure 15 shows the time evolution of the absorbance of four different samples at a temperature of 40 ${ }^{\circ} \mathrm{C}$. At the beginning of the process, all samples appear milky because of the presence of the Carbopol particles dispersed in solution in their initial coiled configuration. Therefore, in the initial stages the light is scattered preventing any transmission through the sample and causing a noisy signal of the absorbance (seen clearly in the black curve in Figure 15). When the solvent starts to penetrate inside the crosslinked particles, creating the swollen equilibrium layer, the core of the coiled particles shrinks causing an exponential decay of the measured absorbance. Similar to the rheological data, an increase in temperature and in the mass fraction of glycerol results in a faster decay of the absorbance. In the slower conditions, the absorbance starts to drop after an induction time, before which the scattering is too large 
to allow any transmission of the incident light. It should be noted that, given the nature of the samples, a further analysis of the measured spectra at the different wavelengths cannot give any additional information. Indeed, the range of wavelengths covered by the UV-vis spectrometer is not appropriate to obtain any information regarding changes in the internal configuration of polymeric mixtures. To this end, other techniques such as FT-IR (Fourier transform infrared) spectroscopy should be implemented [33,51]. Nevertheless, UV-vis spectroscopy can reveal the time needed for the samples to become transparent, which can be used as an indicator for the completion of the dissolution process. Therefore, we calculated a settling time of the absorbance curves for all measured samples as the time required by the normalized absorbance to reduce to $98 \%$ from its initial value. The absorbance signal was normalized considering as maximum the first value after the noisy part of the signal and as minimum the final plateau reached. Characteristic times $\left(t_{s}\right)$ at all conditions are reported in Table 5 together with gelation half-times obtained from the rheological measurements. As can be observed, the qualitative effects of glycerol and temperature are the same, but the settling time predicted with the UV-vis is higher than the characteristic time obtained from the rheological measurements, even if for all temperatures, the difference between the two decreases with the decrease in Carbopol content. For the most concentrated samples, the time scale from the UV-vis measurements is more than twice that from the rheological measurements, while for the most diluted samples the two scales are nearly the same (see SI6 for further details). If we consider that the two methods monitor different aspects of the same phenomenon, this trend confirms the mechanism presented in the previous section. In fact, UV-vis absorbance only tracks the extinction of the dry coiled polymer core, which depends mainly on the diffusion of the solvent into the polymer matrix, while the evolution of the storage modulus also strongly depends on the local interactions between neighbouring swelling particles. The two phenomena, i.e. solvent penetration and chain swelling, proceed 
together in each single particle, but the local interactions between swelling particles arise as soon as the steric effects between neighbours become significant, thus triggering the change in the elastic modulus of the material. Therefore, the two methods considered predict similar time scales when the particle interactions are negligible, i.e. in dilute conditions, which is what is observed experimentally in the results.

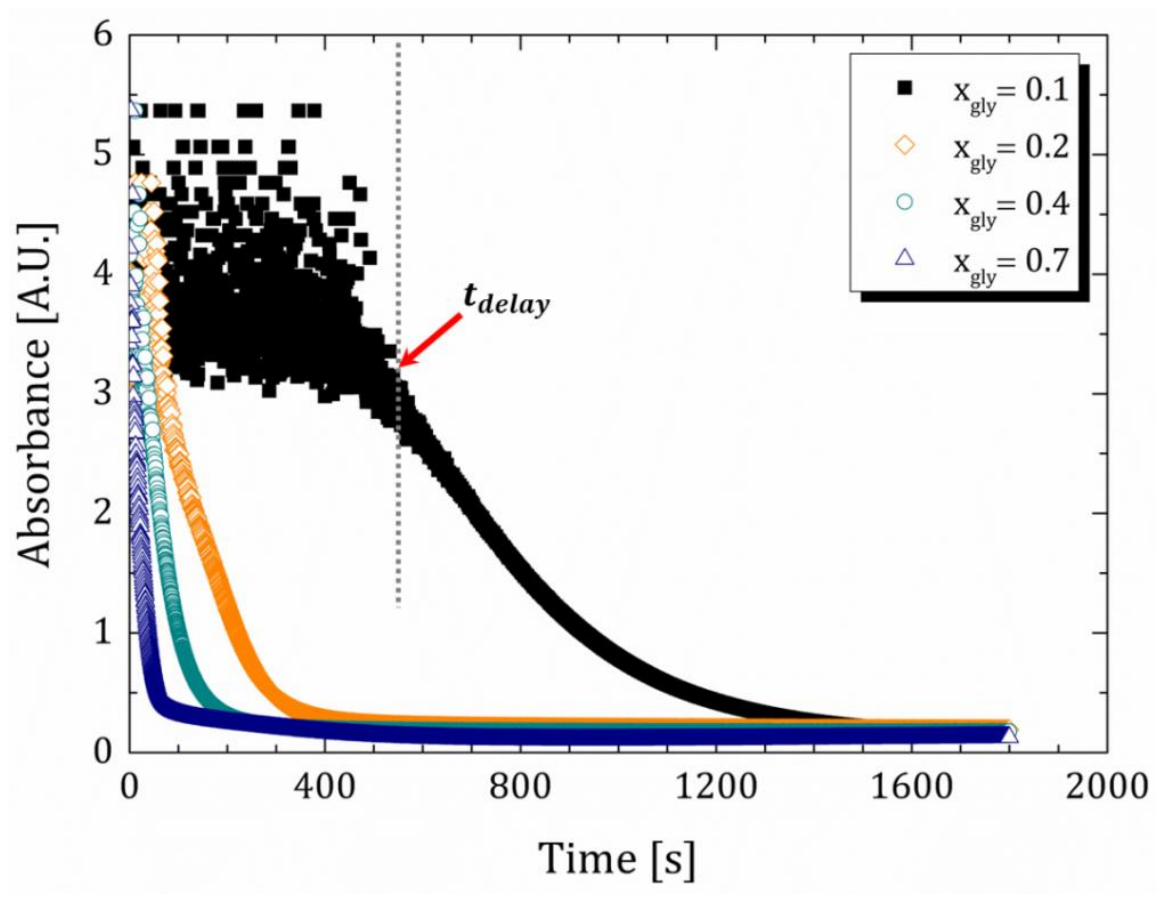

Figure 15 - Time evolution of turbidity (defined as the absorbance at $500 \mathrm{~nm}$ ) during the gelation process of four different samples at $40^{\circ} \mathrm{C}$. At the lower concentration of glycerol the initial scattering of the sample lasts for longer time and the exponential decay is observable only after a certain induction time, as indicated in figure with $t_{\text {delay }}$. 
Table 5 - Settling time of the gelation process, $t_{S}$, calculated from the absorbance measurements for all experimental conditions. The gelation half-times are also reported for direct comparison.

\begin{tabular}{c|cc|cc|cc|cc|cc}
\hline \multirow{2}{*}{ SAMPLE } & \multicolumn{2}{|c|}{ T1 } & \multicolumn{2}{c|}{ T2 } & \multicolumn{2}{c|}{ T3 } & \multicolumn{2}{c|}{ T4 } & \multicolumn{2}{c}{ T5 } \\
\cline { 2 - 11 } & $\boldsymbol{t}_{\boldsymbol{s}}(\boldsymbol{s})$ & $\boldsymbol{\theta}(\boldsymbol{s})$ & $\boldsymbol{t}_{\boldsymbol{s}}(\boldsymbol{s})$ & $\boldsymbol{\theta}(\boldsymbol{s})$ & $\boldsymbol{t}_{\boldsymbol{s}}(\boldsymbol{s})$ & $\boldsymbol{\theta}(\boldsymbol{s})$ & $\boldsymbol{t}(\boldsymbol{s})_{\boldsymbol{s}}$ & $\boldsymbol{\theta}(\boldsymbol{s})$ & $\boldsymbol{t}(\boldsymbol{s})_{\boldsymbol{s}}$ & $\boldsymbol{\theta}(\boldsymbol{s})$ \\
\cline { 2 - 11 } $\mathbf{X 1}$ & $>3500$ & 3385 & $>3500$ & 1242 & 1536 & 379 & 556 & 277 & 235 & 163 \\
$\mathbf{X 2}$ & 3485 & 1538 & 2714 & 912 & 456 & 202 & 212 & 133 & 133 & 89 \\
$\mathbf{X 3}$ & 2272 & 1201 & 916 & 397 & 411 & 156 & 183 & 84 & 112 & 72 \\
$\mathbf{X 4}$ & 1440 & 955 & 873 & 253 & 383 & 103 & 95 & 64 & 106 & 51 \\
$\mathbf{X 5}$ & 994 & 881 & 521 & 307 & 297 & 90 & 109 & 58 & 73 & 41 \\
$\mathbf{X 6}$ & 909 & 778 & 476 & 256 & 198 & 88 & 97 & 52 & 57 & 38 \\
$\mathbf{X 7}$ & 813 & 762 & 437 & 200 & 151 & 63 & 94 & 41 & 49 & 28 \\
$\mathbf{X 8}$ & 771 & 656 & 225 & 216 & 97 & 48 & 55 & 30 & 43 & 23 \\
\hline
\end{tabular}

\subsection{Application of the kinetic equation: Conversion maps}

From what we observed so far, the kinetic behaviour of the gelling mixtures of our interest is highly dependent on both operating temperature and solvent composition. The dramatic rheological change that the material undergoes during the gelation process can have significant consequences on the efficiency of the process itself and therefore on the properties of the final product obtained. Hence, the kinetic equation of the structural conversion degree (Eq. (6), along with Eqs. (7)-(9)) can be used to obtain conversion maps which could potentially be employed to design new manufacturing processes involving materials used in this work. The maps have been obtained at a constant temperature and they report the conversion degree $X_{S}$ for the full range of sample compositions against a process residence time. The maps at three temperatures $\left(25,40\right.$ and $\left.60{ }^{\circ} \mathrm{C}\right)$ are reported in Figure 16. The colour legend shows the evolution of the structural conversion degree with the residence time (horizontal axis) and the glycerol mass fraction (vertical axis). To obtain a smooth map for 
$X_{S}$, in Eq. (6) we replaced the parameters $\theta$ and $n$ with their fitting equations; specifically, for the gelation half-time we used Eq. (8), while for the coefficient $n$, by combining Eqs. (7), (8) and (9), we obtained the following relation:

$$
n\left(x_{g l y}\right)=4 k_{P} k_{\theta}\left(x_{g l y}\right)^{\left(m_{p}-m_{\theta}\right)}
$$

As can be observed in Figure 16, the qualitative trend is the same for the three temperatures: at lower mass fractions of glycerol the transition is slower, while for higher mass fractions the boundaries of the maps collapse to a vertical asymptote, showing (as expected) a faster transition. Therefore, the change in operating temperature simply shrinks or expands the time of the whole process. This aspect can be useful when the whole manufacturing process presents different stages (e.g., mixing, gelation, inclusion of additives), which, with the help of the conversion maps, could be designed in a modular way, by changing the temperature to slow down or accelerate the gelation process. The simplest example is the mixing of the two liquid phases. In this case, it would be advisable to avoid during the mixing process any gelation of the material that increases viscosity; this would ensure a complete homogeneity of the final gel matrix. To this end, the mixing section should have a residence time lower than the time needed to obtain an appreciable change of the structural degree of conversion. To achieve this, it is convenient to work at a temperature sufficiently low as to render the gelation process slow enough. Hence, the conversion maps just derived can offer a first guidance in the mixer design by allowing to determine the required temperature range wherein the system should work; however, it is necessary to highlight that the maps should be used with caution for processes in flow, given the possible influence that the shear history could have on the kinetics of gelling systems. 

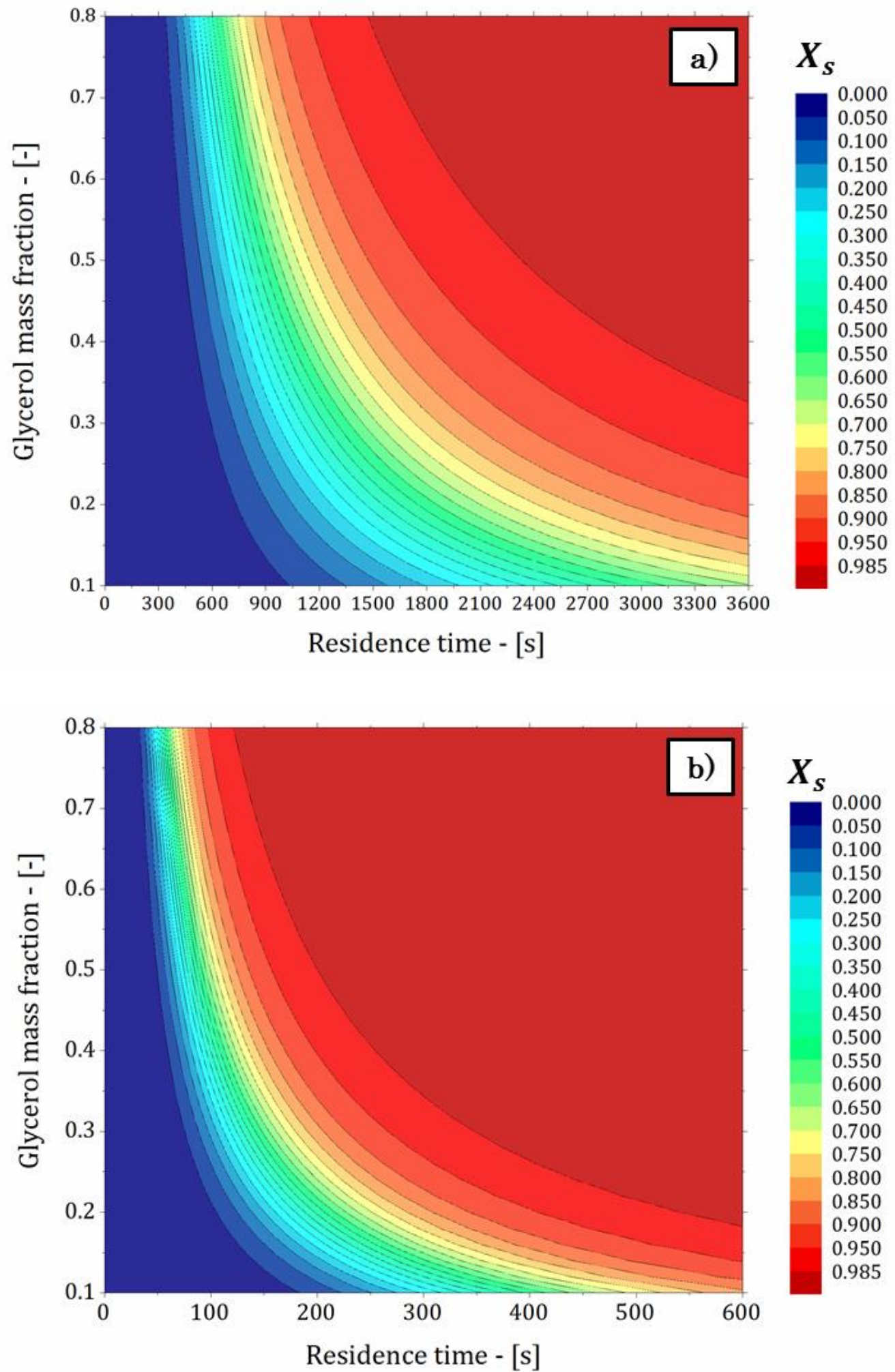


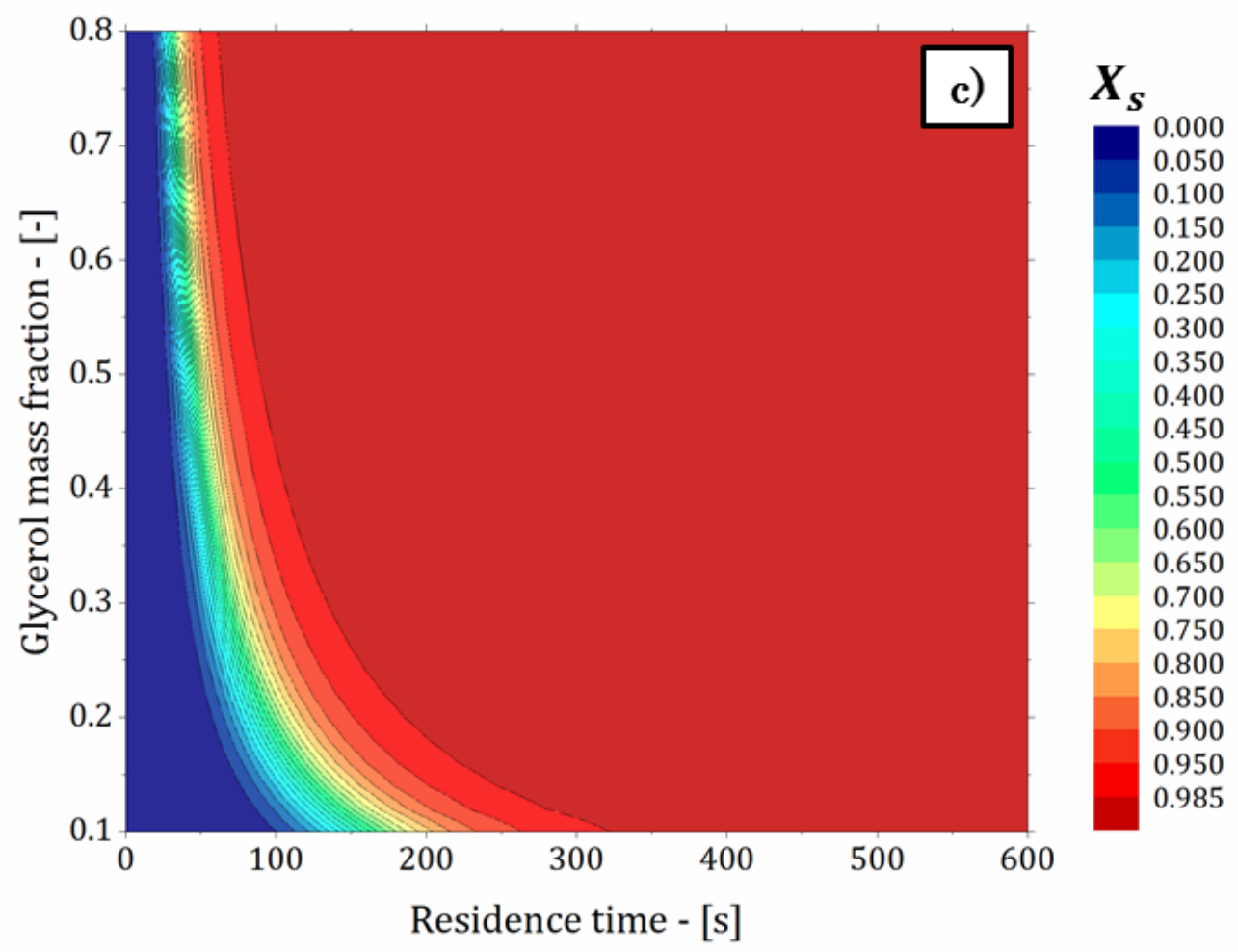

Figure 16 - Conversion maps reporting the structural conversion degree $\boldsymbol{X}_{\boldsymbol{S}}$ against residence time and glycerol mass fraction at a temperature of a) $\left.25^{\circ} \mathrm{C}, \boldsymbol{b}\right) 40^{\circ} \mathrm{C}$ and c) $60^{\circ} \mathrm{C}$.

\section{Conclusions}

A kinetic analysis of the gelation process of non-aqueous Carbopol dispersions in PEG400 and glycerol has been performed by means of FTMS and UV-vis spectroscopy. The structure evolution of different samples has been tracked in time with focus on the effect of gelation temperature and dilution with different solvent concentrations. Both temperature and composition of the solvent have a dramatic effect on the kinetics, where an increase in both temperature and mass fraction of the solvent with higher polarity and small molecular dimension (glycerol) generates an increase in the characteristic rate of the process. A simple Fickian diffusive mechanism, based on polymer dissolution theory, has been used to explain the trends observed. UV-vis spectroscopy has also been implemented to confirm the dominance of the diffusive phenomenon. 
From the final comparison between the characteristic times of the two experimental methods, it is deducted that both monitor two different aspects of the mechanism: through the observed absorbance decay, it is possible to track the characteristic time of the solvent penetration inside the cross-linked Carbopol structure, while, the time evolution of the storage modulus gives a more complete understanding of the internal microstructure changes, which depend not only on the solvent diffusion kinetics into the cross-linked polymer but also on the local interactions between swelling particles. Thus, from a process design viewpoint UV-vis spectroscopy can give an estimation of dry core extinction time. However, if the time evolution of the material structure is essential for design purposes, purely optical techniques based on turbidity could be misleading and a direct measurement of the structure evolution should be carried out.

Finally, we exploited the kinetic equation defined in Eq. (6) together with the equations for the two fitting parameters (Eqs. (8) and (9)), to summarise in conversion maps the overall time evolution of the gelation process at different operating conditions. Naturally, the presented maps are specific for the system studied. Our intention, however, was to show the relevance of the kinetic analysis performed to processing and to develop a generic methodology that can be applied to other systems where the change in rheological properties affects the design of the process. 


\section{Acknowledgements}

The authors would like to acknowledge GlaxoSmithKline Consumer Healthcare and the EPSRC (EP/N024915/1) for the financial support given to this project. 


\section{References}

[1] J.D. Ferry, Viscoelastic properties of Polymers, 3rd ed., John Wiley \& Sons, Inc, New York, 1980.

[2] C. Zhao, G. Yuan, D. Jia, C.C. Han, Macrogel induced by microgel: Bridging and depletion mechanisms, Soft Matter. 8 (2012) 7036-7043. doi:10.1039/c2sm25409c.

[3] B.W. Barry, M.C. Meyer, Rheological properties of carbopol gels: 1. Continuous shear and creep properties of carbopol gels, Int. J. Pharm. 2 (1979) 1-25.

[4] J.Y. Kim, J.Y. Song, E.J. Lee, S.K. Park, Rheological properties and microstructures of Carbopol gel network system, Colloid Polym. Sci. 281 (2003) 614-623. doi:10.1007/s00396-002-0808-7.

[5] B.W. Barry, M.C. Meyer, The rheological properties of carbopol gels 2. Oscillatory properties of carbopol gels., Int. J. Pharm. 2 (1979) 27-40.

[6] N.W. Taylor, E.B. Bagley, Dispersion or Solutions? A mechanism for Certain Thickening Agents, J. Appl. Polym. Sci. 18 (1974) 2747-2761. doi:10.1002/app.1974.070180916.

[7] S.J. Curran, R.E. Hayes, A. Afacan, M.C. Williams, P.A. Tanguy, Properties of carbopol solutions as models for yield-stress fluids, J. Food Sci. 67 (2002) 176-180. doi:10.1111/j.1365-2621.2002.tb11379.x.

[8] R.J. Ketz, R.K. Prud'homme, W.W. Graessley, Rheology of concentrated microgel solutions, Rheol. Acta. 27 (1988) 531-539. doi:10.1007/BF01329353.

[9] J.M. Piau, Carbopol gels: Elastoviscoplastic and slippery glasses made of individual swollen sponges. Meso- and macroscopic properties, constitutive equations and scaling laws, J. Nonnewton. Fluid Mech. 144 (2007) 1-29. doi:10.1016/j.jnnfm.2007.02.011.

[10] J.S. Chu, D.M. Yu, G.L. Amidon, N.D. Weiner, A.H. Goldberg, Viscoelastic Properties of Polyacrylic Acid Gels in Mixed Solvents, Pharm. Res. 9 (1992) 16591663. doi:10.1023/A:1015841214591.

[11] G. Bonacucina, S. Martelli, G.F. Palmieri, Rheological, mucoadhesive and release properties of Carbopol gels in hydrophilic cosolvents, Int. J. Pharm. 282 (2004) 115130. doi:10.1016/j.ijpharm.2004.06.012.

[12] M. Dinkgreve, M. Fazilati, M.M. Denn, D. Bonn, Carbopol: From a simple to a thixotropic yield stress fluid, J. Rheol. (N. Y. N. Y). 62 (2018) 773-780. doi:10.1122/1.5016034.

[13] W. De Carvalho, M. Djabourov, Physical gelation under shear for gelatin gels, Rheol. 
Acta. 36 (1997) 591-609. doi:10.1007/BF00367355.

[14] I.T. Norton, D.A. Jarvis, T.J. Foster, A molecular model for the formation and properties of fluid gels, Int. J. Biol. Macromol. 26 (1999) 255-261. doi:10.1016/S0141-8130(99)00091-4.

[15] I. Fernández Farrés, I.T. Norton, Formation kinetics and rheology of alginate fluid gels produced by in-situ calcium release, Food Hydrocoll. 40 (2014) 76-84. doi:10.1016/j.foodhyd.2014.02.005.

[16] M. Yonese, Sustained drug delivery by gels, in: Y. Osada, K. Kajiwara (Eds.), Gels Handb., Academic Press, San Diego, 2001: pp. 230-240. doi:10.1016/B978012394690-4/50115-8.

[17] A.K. Singla, M. Chawla, A. Singh, Potential Applications of Carbomer in Oral Mucoadhesive Controlled Drug Delivery System: A Review, Drug Dev. Ind. Pharm. 26 (2010) 913-924. doi:10.1081/DDC-100101318.

[18] P. Lefrançois, E. Ibarboure, B. Payré, E. Gontier, J.F. Le Meins, C. Schatz, Insights into Carbopol gel formulations: Microscopy analysis of the microstructure and the influence of polyol additives, J. Appl. Polym. Sci. 132 (2015). doi:10.1002/app.42761.

[19] H.H. Winter, F. Chambon, Analysis of Linear Viscoelasticity of a Crosslinking Polymer at the Gel Point, J. Rheol. (N. Y. N. Y). 30 (1986) 367-382. doi:10.1122/1.549853.

[20] M.E. De Rosa, H.H. Winter, The effect of entanglements on the rheological behavior of polybutadiene critical gels, Rheol. Acta. 33 (1994) 220-237. doi:10.1007/BF00437307.

[21] M. Mours, H.H. Winter, Relaxation patterns of nearly critical gels, Macromolecules. 29 (1996) 7221-7229. doi:10.1021/ma9517097.

[22] E.E. Holly, S.K. Venkataraman, H.H. Winter, F. Chambon, Fourier Transform Mechanical Spectroscopy of Viscoelastic Materials With Transient Structure, J. Nonnewton. Fluid Mech. 27 (1988) 17-26. doi:10.1016/0377-0257(88)80002-8.

[23] M. Wilhelm, Fourier-transform rheology, Rheol. Acta. 37 (1998) 399-405. doi:doi.org/10.1007/s003970050126.

[24] A. Ponton, S. Barboux-Doeuff, C. Sanchez, Rheology of titanium oxide based gels: Determination of gelation time versus temperature, Colloids Surfaces A Physicochem. Eng. Asp. 162 (2000) 177-192. doi:10.1016/S0927-7757(99)00249-6.

[25] D.J. Curtis, A. Holder, N. Badiei, J. Claypole, M. Walters, B. Thomas, M. Barrow, D. Deganello, M.R. Brown, P.R. Williams, K. Hawkins, Validation of Optimal Fourier 
Rheometry for rapidly gelling materials and its application in the study of collagen gelation, J. Nonnewton. Fluid Mech. $222 \quad$ (2015) 253-259. doi:10.1016/j.jnnfm.2015.01.003.

[26] E.O. Brigham, The Fast Fourier Transform, IEEE Spectr. 4 (1967) 67-70. doi:10.1109/MSPEC.1967.5217220.

[27] C.W. Macosko, Rheology: Principles, Measurements and Applications, Wiley-VCH, Inc, 1994.

[28] J. Hofrichter, P.D. Ross, W.A. Eaton, Kinetics and Mechanism of Deoxyhemoglobin S Gelation: A New Approach to Understanding Sickle Cell Disease, Proc. Natl. Acad. Sci. 71 (1974) 4864-4868. doi:10.1073/pnas.71.12.4864.

[29] M.K. Bain, M. Bhowmik, S.N. Ghosh, D. Chattopadhyay, In Situ Fast Gelling Formulation of Methyl Cellulose for In Vistro Ophtalmic Controlled Delivery of Ketorolac Tromethamine, J. Appl. Polym. Sci. 113 (2009) 1241-1246. doi:10.1002/app.30040.

[30] M.K. Bain, M. Bhowmik, D. Maity, N.K. Bera, S. Ghosh, D. Chattopadhyay, Control of Thermo Reversible Gelation of Methylcellulose Using Polyethylene Glycol and Sodium Chloride for Sustained Delivery of Ophthalmic Drug, J. Appl. Polym. Sci. 118 (2010) 631-637. doi:10.1002/app.32350.

[31] N.W. Taylor, S.H. Gordon, Shear Modulus in Closely Packed Gel Suspension, J. Appl. Polym. Sci. 27 (1982) 4377-4386. doi:10.1002/app.1982.070271127.

[32] A. Gabriele, F. Spyropoulos, I.T. Norton, Kinetic study of fluid gel formation and viscoelastic response with kappa-carrageenan, Food Hydrocoll. 23 (2009) 2054-2061. doi:10.1016/j.foodhyd.2009.03.018.

[33] M.T. Islam, N. Rodríguez-Hornedo, S. Ciotti, C. Ackermann, Fourier transform infrared spectroscopy for the analysis of neutralizer-carbomer and surfactant-carbomer interactions in aqueous, hydroalcoholic, and anhydrous gel formulations, AAPS J. 6 (2004) 61-67. doi:10.1208/aapsj060435.

[34] E. Weber, M. Moyers-González, T.I. Burghelea, Thermorheological properties of a Carbopol gel under shear, J. Nonnewton. Fluid Mech. 183-184 (2012) 14-24. doi:10.1016/j.jnnfm.2012.07.005.

[35] J. Janacek, J.D. Ferry, Viscoelastic properties of cross-linked solutions of poly(betahydroxyethyl methacrylate) in diethylee glycol I. Creep and equilibrium compliance measurements, Rheol. Acta. 9 (1970) 208-218.

[36] C.-P. Wong, J.L. Schrag, J.D. Ferry, Molecular Mobility in Swollen Cross-linked 
Rubbers. Viscoelasticity and Diffusion Measurements, Polym. J. 2 (1971) 274-282.

[37] J.R. Stokes, W.J. Frith, Rheology of gelling and yielding soft matter systems, Soft Matter. 4 (2008) 1133-1140. doi:10.1039/b719677f.

[38] T.S.K. Ng, G.H. McKinley, Power law gels at finite strains: The nonlinear rheology of gluten gels, J. Rheol. (N. Y. N. Y). 52 (2008) 417-449. doi:10.1122/1.2828018.

[39] Y.G. Lin, D.T. Mailin, J.C.W. Chien, H.H. Winter, Dynamic Mechanical Measurement of Crystallization-Induced Gelation in Thermoplastic Elastomeric Poly(propylene), Macromolecules. 24 (1991) 850-854.

[40] M. Jokinen, E. Gyorvary, J.B. Rosenholm, Viscoelastic characterization of three different sol - gel derived silica gels, Colloids Surfaces A Physicochem. Eng. Asp. 141 (1998) 205-216.

[41] M. Verheul, S.P.F.M. Roefs, J. Mellema, Power Law Behavior of Structural Properties of Protein Gels, 7463 (1998) 2263-2268. doi:10.1021/la9708187.

[42] S.M. Magami, R.L. Williams, Gelation studies on acrylic acid-based hydrogels via in situ photo-crosslinking and rheology, J. Appl. Polym. Sci. 135 (2018) 46691. doi:10.1002/app.46691.

[43] D. Calvet, J.Y. Wong, S. Giasson, Rheological monitoring of polyacrylamide gelation: Importance of cross-link density and temperature, Macromolecules. 37 (2004) 77627771. doi:10.1021/ma049072r.

[44] J. Giraldo, N.M. Vivas, E. Vila, A. Badia, Assessing the (a)symmetry of concentration-effect curves: Empirical versus mechanistic models, Pharmacol. Ther. 95 (2002) 21-45. doi:10.1016/S0163-7258(02)00223-1.

[45] B.A. Miller-Chou, J.L. Koenig, A review of polymer dissolution, Prog. Polym. Sci. 28 (2003) 1223-1270. doi:10.1016/S0079-6700(03)00045-5.

[46] A. Messaâdi, N. Dhouibi, H. Hamda, F.B.M. Belgacem, Y.H. Adbelkader, N. Ouerfelli, A.H. Hamzaoui, A New Equation Relating the Viscosity Arrhenius Temperature and the Activation Energy for Some Newtonian Classical Solvents, J. Chem. 2015 (2015) 7-10. doi:10.1155/2015/163262.

[47] E. Kaunisto, M. Marucci, P. Borgquist, A. Axelsson, Mechanistic modelling of drug release from polymer-coated and swelling and dissolving polymer matrix systems, Int. J. Pharm. 418 (2011) 54-77. doi:10.1016/j.ijpharm.2011.01.021.

[48] Y.-O. Tu, A.C. Ouano, Model for the Kinematics of Polymer Dissolution, IBM J. Res. Dev. 21 (1977) 131-142. doi:10.1147/rd.212.0131.

[49] I. Devotta, V.D. Ambeskar, A.B. Mandhare, R.A. Mashelkar, The life time of a 
dissolving polymeric particle, Chem. Eng. Sci. 49 (1994) 645-654. doi:10.1016/00092509(94)85010-0.

[50] I. Devotta, R.A. Mashelkar, Role of Thermodynamic and Kinetic Factors in Polymer Dissolution in Mixed Solvents+, Chem. Eng. Commun. 156 (2010) 31-43. doi:10.1080/00986449708936667.

[51] M.K. Jaiswal, R. Banerjee, P. Pradhan, D. Bahadur, Thermal behavior of magnetically modalized poly(N-isopropylacrylamide)-chitosan based nanohydrogel, Colloids Surfaces B Biointerfaces. 81 (2010) 185-194. doi:10.1016/j.colsurfb.2010.07.009. 